\title{
Recent advances in developing optical and electrochemical sensors for analysis of methamphetamine: A review
}

\author{
Zeynab Khorablou ${ }^{\text {a }}$, Faezeh Shahdost-fard ${ }^{b}$, Habib Razmi ${ }^{\text {a, }}{ }^{* *}$, Mehmet Lütfi Yola ${ }^{\text {, }}$ \\ Hassan Karimi-Maleh d, e, f, * \\ a Department of Chemistry, Faculty of Basic Sciences, Azarbaijan Shahid Madani University, PO BOX 53714-161, Tabriz, Iran \\ ${ }^{\mathrm{b}}$ Department of Science, Farhangian University, Tehran, 19396-14464, Iran \\ ${ }^{\mathrm{c}}$ Hasan Kalyoncu University, Faculty of Health Sciences, Department of Nutrition and Dietetics, Gaziantep, Turkey \\ ${ }^{\mathrm{d}}$ School of Resources and Enviroment, University of Electronic Science and Technology of China, P.O. Box 611731, Xiyuan Ave, Chengdu, PR China \\ e Department of Chemical Engineering, Quchan University of Technology, Quchan, 9477177870, Iran \\ ${ }^{\mathrm{f}}$ Department of Chemical Sciences, University of Johannesburg, Doornfontein Campus, 2028, Johannesburg, P.O. Box 17011, South Africa
}

\section{H I G H L I G H T S}

- The origin of MAMP and its side effects have been reported.

- The optical and electrochemical sensors for sensing MAMP have been reviewed.

- The advantages and drawbacks of the applied modifiers and interfaces have been described.

- The undeniable role of nanotechnology in the expansion of the MAMP sensors has been described.

- Some offers for commercializing of MAMP sensors for the rutin analysis with low cost have been proposed.

\section{A R T I C L E I N F O}

\section{Article history:}

Received 31 December 2020

Received in revised form

10 March 2021

Accepted 25 March 2021

Available online 29 March 2021

Handling Editor: Derek Muir

\section{Keywords:}

Methamphetamine

Electrochemical sensors

Optical sensors

Illicit drug
G R A P H I C A L A B S T R A C T

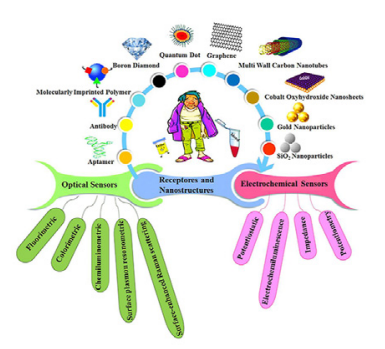

\begin{abstract}
A B S T R A C T
Recognition of misused stimulant drugs has always been a hot topic from a medical and judicial perspective. Methamphetamine (MAMP) is an addictive and illegal drug that profoundly affects the central nervous system. Like other illicit drugs, the detection of MAMP in biological and street samples is vital for several organizations such as forensic medicine, anti-drug headquarters and diagnostic clinics. By emerging nanotechnology and exploiting nanomaterials in sensing applications, a great deal of attention has been given to the design of analytical sensors in MAMP tracing. For the first time, this study has briefly reviewed all the optical and electrochemical sensors in MAMP detection from earlier so far. How various receptors with engineering nanomaterials allow developing novel approaches to measure MAMP have been studied. Fundamental concepts related to optical and electrochemical recognition assays in which nanomaterials have been used and relevant MAMP sensing applications have been
\end{abstract}

* Corresponding author. School of Resources and Enviroment, University of Electronic Science and Technology of China, P.O. Box 611731, Xiyuan Ave, Chengdu, PR China.

** Corresponding author.

E-mail addresses: h.razmi@azaruniv.ac.ir, mh_razmi@yahoo.com (H. Razmi), hassan@uestc.edu.cn (H. Karimi-Maleh). 


\section{Introduction}

Recently, the changing narcotic use patterns from traditional to industrial chemical forms and psychotropic drugs is a great alarm for society (Karimi-Maleh et al., 2021a). Methamphetamine (MAMP), N-methyl-1-phenylpropane-2-amine, is one of the famous industrial opiates, known as the second most widely used illicit drug worldwide after cannabis (Shakeri et al., 2019; Won et al., 2013). MAMP has been firstly produced from ephedrine (Rusyniak 2013) and now illegally synthesized in underground laboratories to misuse through oral, inhaled, injectable, and smoked forms (Anglin et al., 2000; Karila et al., 2010). The worldwide synthesis of amphetamine-type stimulants (ATS) is approximately 500 metric tons a year, with 24.7 million abusers, according to the data United Nations Office on Drugs and Crime (UNODC) (Adams et al., 2016). Although, in some cases, MAMP as a drug is prescribed in low dosage to treat many disorders, including hyperactivity disorder, narcolepsy and excessive obesity (Prakash et al., 2017). In contrast, this popular street opiate has been broadly used as a recreational stimulant substance to create a feeling of extreme pleasure in youth and teenagers. By blocking serotonin, dopamine and norepinephrine receptors in the brain, this neurotoxic substance causes irreparable problems (Parsons 2014). Taking high doses of MAMP leads to violent behaviors, disturbance of consciousness, paranoia and meth mouth (Donaldson and Goodchild 2006; Prakash et al., 2017; Shaner et al., 2006; Wearne and Cornish 2018). The increased heart rate and blood pressure and respiratory problems in MAMP consumers may lead to stroke and death (Mooney et al., 2009). The schematic representation of the side effects of this stimulant drug is shown in
Scheme 1 with detail. To control the epidemic problems of MAMP abuse, developing a reliable method for on-site screening of human biofluids and seized ecstasy drugs is essential for the legal and medical authorities. This global challenge has encouraged many researchers to develop various analytical approaches in early MAMP detection through different methods. The gas chromatography (GC) (Djozan et al., 2012) liquid chromatography (LC) (Chou and Lee 2005) and high performance liquid chromatography (HPLC) (Kumihashi et al., 2007) are traditional analytical methods for the detection of the MAMP and derivatives especially when combined with other methods including supercritical fluid chromatography (SFC)/tandem mass spectrometry (MS-MS) (Segawa et al., 2021), liquid chromatography-tandem mass spectrometry (LC-MS-MS) (Kahl et al., 2021). Except for the chromatographic methods, quartz crystal microbalance (QCM) (Miura et al., 1993), ion mobility spectrometry (IMS) (Alizadeh et al., 2008), capillary GC (Tanaka et al., 1994) and capillary electrophoresis (CE) (Chinaka et al., 2000) have been also reported for the MAMP measuring with high sensitivity and accuracy. Although these methods are valid for the MAMP tracing in the complex matrixes, some drawbacks still exist, such as the elaborate sample preparation and timeconsuming, expensive instruments and trained personnel. Other methods in MAMP detection are based on optically and electrochemically strategies. Our motivation in this review is a general overview of the design principles and fundamental features of the MAMP sensors, from the great variety of the applied nanostructures and biocaptures to various sensing mechanisms (Scheme 2). Accordingly, various optical and electrochemical approaches published in the past two decades have been summarized to discuss in detail. Finally, the remaining challenges and future directions on

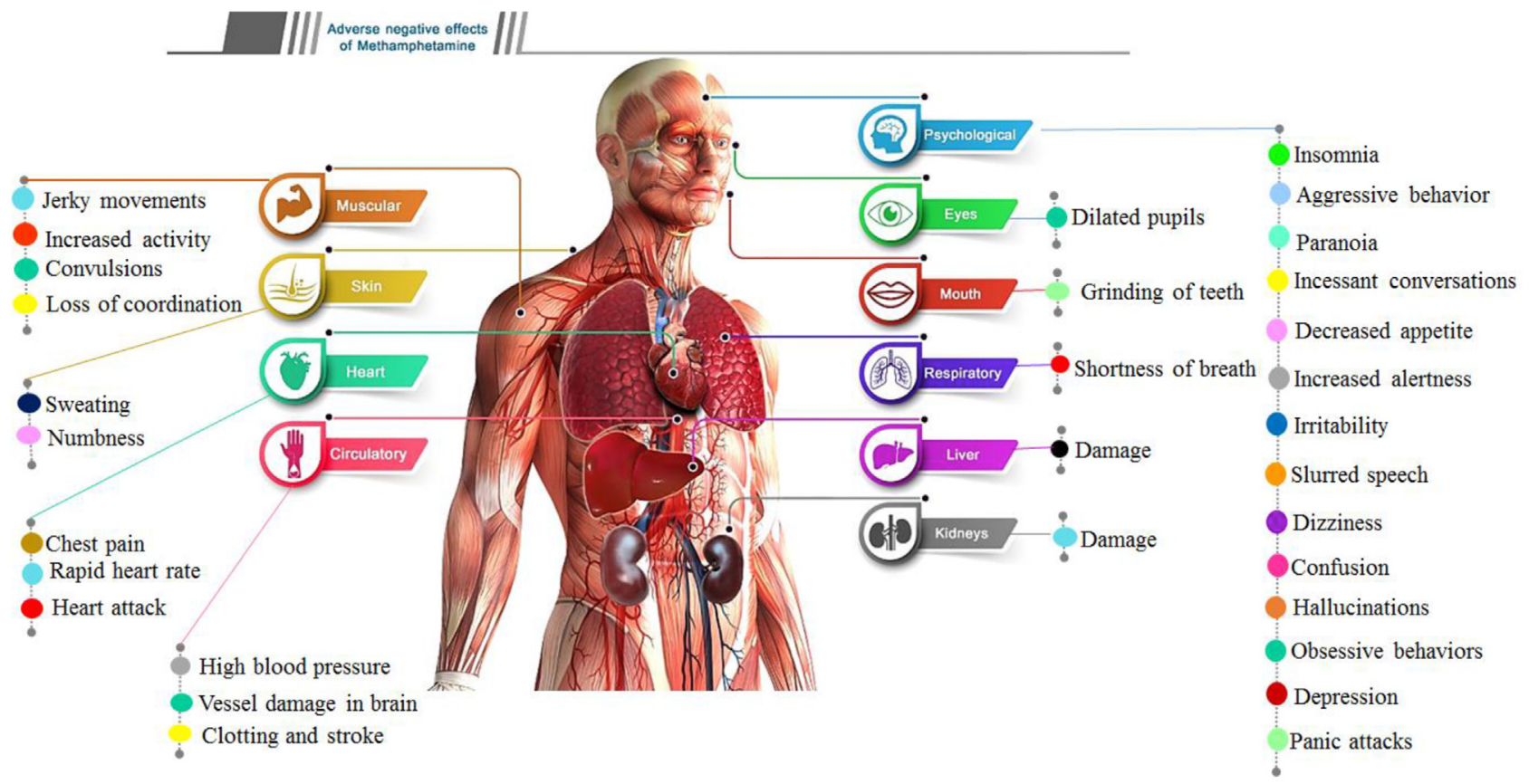

Scheme 1. Side effects of MAMP use in different parts of the human body. 


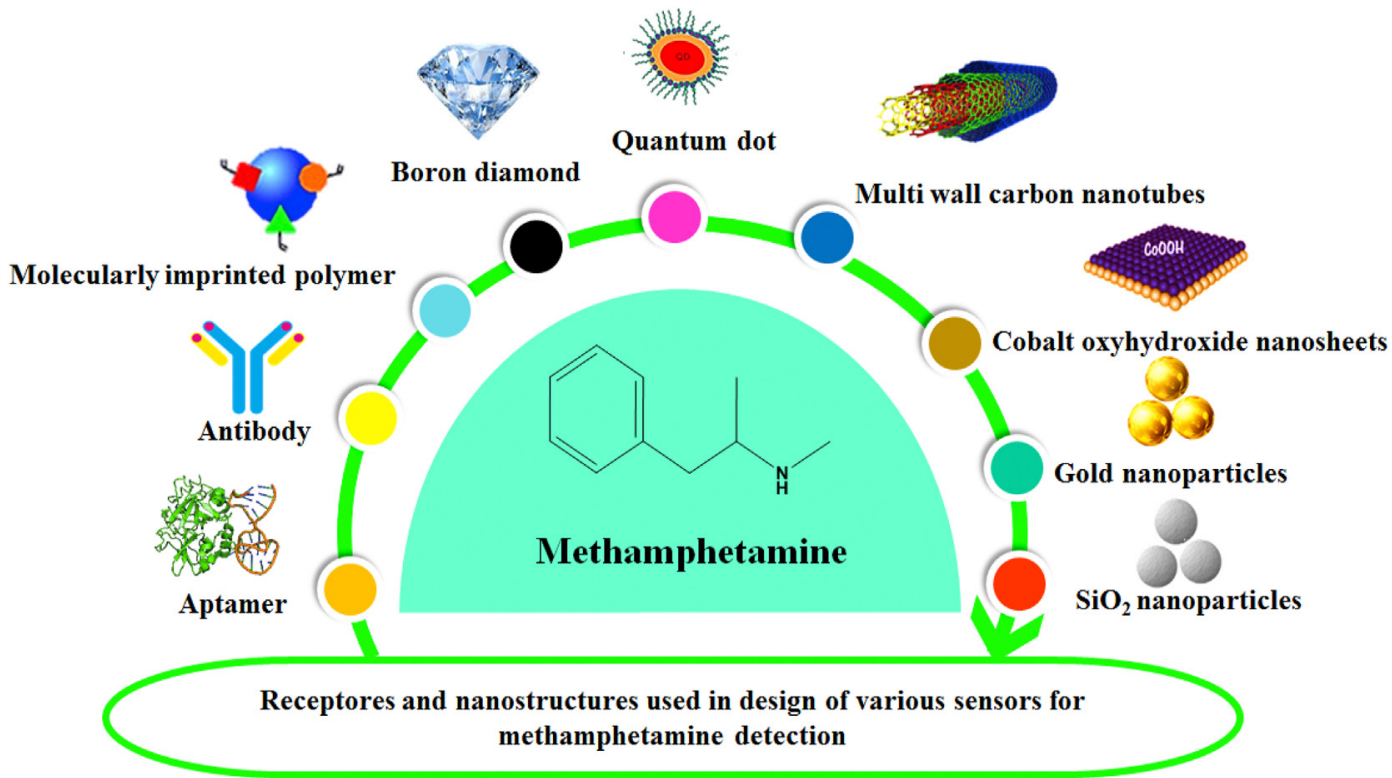

Scheme 2. Receptors and nanostructures used in the design of various sensors for MAMP detection.

MAMP sensing have been highlighted.

\section{Optical sensors in MAMP detection}

Optical sensors have been considered functional tools because of their remarkable ability to exhibit the interactions between the substances and sensor elements to provide analyte information through a simple and low-cost route (Lobnik et al., 2012; Pan et al., 2013). Generally, the optical sensors consist of a transducer that captures signal from a phenomenon and turns them into different radiations. It provides diverse optical sensing strategies, namely, fluorescence, colorimetry, chemiluminescence, surface plasmon resonance (SPR) and surface-enhanced Raman scattering (SERS) approaches (Scheme 3) (Pashazadeh et al., 2017). In the following, different types of optical sensing strategies in MAMP detection are briefly reported.

\subsection{Fluorimetric sensors}

By emission light of an excited molecule upon returning to its ground state, the fluorescence (FL) is occurred (Skoog et al., 2017). Since some molecules have this property, so this method is inherently selective. The naked-eye detection of interaction with easy access in quantitative analysis, high sensitivity, wide response range and rapid analysis are advantages of the fluorescence-based sensors (Dandin et al., 2007; Pickup et al., 2005).

The first fluorimetric sensor in trace measurement of MAMP was been proposed by He et al., in 2011. They applied a polyfluorene

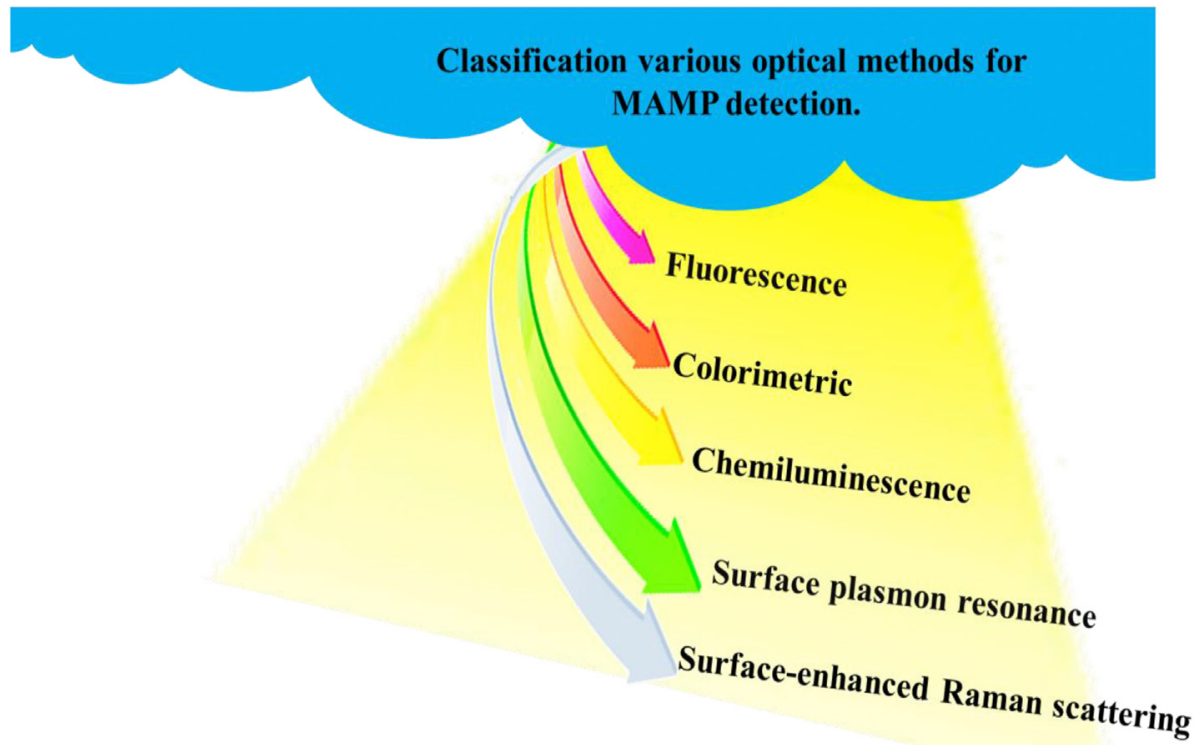

Scheme 3. Classification of various optical methods of the MAMP sensor. 
with the $\mathrm{NH}_{2}$-terminated side chains as a fluorescent probe and by injection of MAMP to the system, the FL spectra were switched off. This turn-on-off system detected MAMP with a limit of detection (LOD) of $178.2 \mathrm{nM}$ (Cheng et al., 2011).

Wen's group presented another study based on the coupled polymer structures in detecting MAMP vapor in 2012. They displayed the sensing performance and the photostability benchmark of three polymer films including P1 (poly[(9,9-dioctylfluorenyl-2,7diyl)-alt(2,1,3-benzothiadiazole-4,7-diyl)]), P2 (poly[(9,9dioctylfluorenyl-2,7-diyl)-alt(2,1,3-benzooxadiazole-4,7-diyl)])

and P3 (poly[(9,9-dioctylfluorenyl-2,7-diyl)-alt(quinoxaline-5,8diyl)]) which were exposed to saturated MAMP vapor. The results indicated the P1 polymer was highly sensitive and selective regards to MAMP in such that a LOD of $1.283 \mu \mathrm{M}$ (Wen et al., 2012).

A more in-deep study of the fluorimetric methods reflects that nanotechnology appearance has a profound effect on MAMP measurement over the years and opened new horizons for signaling assistance in some sensors. Among various nanomaterials, silica nanoparticles (SiO2NPs) is an admirable probe in optical sensing due to several excellent features such as nontoxic, optical transparency and the freely available natural source (Si 2015). Accordingly, Rouhani and Haghgoo in 2015 reported a fluorimetric method by using a 1,8-naphthalimide-thiophene (NTS) doped $\mathrm{SiO}_{2} \mathrm{NPs}$ (NTS@SiO ${ }_{2} \mathrm{NPs}$ ) for MAMP detection. They showed that incorporating NTS in the SiO2NPs causes significant amelioration of the FL response by increasing MAMP in both aqueous and solid states. The turn-on sensing mechanism related to the weak bonding of heavy sulfur atoms strengthened the spin-orbit coupling and improved the intersystem crossing transitions from $\mathrm{S} 1 \rightarrow \mathrm{T} 1$. The binding of the amines of MAMP to the thiophene unit decreased the internal heavy atom effect and enhanced the FL signal. This sensor detected MAMP in a linear range of 6.7-270 $\mu \mathrm{M}$ (Rouhani and Haghgoo 2015).

Compared to the classic fluorescent organic dyes, quantum Dots (DQs), as the efficient semiconductor nanoparticles, have been used as excellent nano-fluore tags in various optosensing. They exhibit unique chemical and optical highlights, including high quantum yield, great symmetric, narrow emission band, broad absorption spectra and preferable biocompatibility due to their nanoscale dimensions (Dennis et al., 2012; Dubertret et al., 2002; Gao et al., 2004; Mansur et al., 2011; Meijerink 2008; Mohammad-Rezaei and Razmi 2012; Roushani and Shahdost-fard 2017, 2019; Ruedas-Rama et al., 2012; Shahdost-fard et al.). Masteri Farahani et al. took advantage of cadmium sulfide QDs (CdS QDs) as a platform for the antibody immobilization as a bioreceptor of MAMP. The covalent conjugation of mercaptoacetic acid capped CdS QDs with anti-MAMP in the presence of N-ethyl- $\mathrm{N}^{\prime}-(3-$ dimethylaminopropyl carbodiimide) (EDC) and Sulfo- $N$-hydroxysuccinimide (Sulfo-NHS) as coupling agents was achieved. This optosensor presented an excellent capability in the sensitive detection of MAMP under a fluorescence resonance energy transfer (FRET) mechanism with a LOD of $72.78 \mathrm{nM}$ (Fig. 1). Some interference drugs such as codeine and ibuprofen without significant signal confirmed the sensor selectivity (Masteri-Farahani and Mosleh 2019).

However, antibody-based sensors (immunosensors) render many advantages but some disadvantages such as high cost, short shelf-life and hard-availability limit the scope of construction of these sensitive sensors in MAMP detection. To overcome this problem, recently, molecularly imprinted polymers (MIPs) have been introduced as another receptor of MAMP to create binding site templates in synthetic polymers using molecular imprints or patterns(Karimi-Maleh et al., 2021b). Since the MIPs present some valuable features such as low cost, acceptable selectivity, reusability and easy preparation process, they are chosen for the sensor fabricate (Guoning et al., 2019; Haupt 2001; Haupt and Mosbach 2000). Graphene quantum dots (GQDs), as another nano-fluore tag, is a fantastic probe with amazing optical properties (Razmi and Mohammad-Rezaei 2013; Shen et al., 2011). This nanostructure plays an important role in the development optosensors due to excellent quantum yield, the large surface to volume ratio and good biocompatibility (Shen et al., 2012). A combination of MIPs and GQDs as a signal amplifier offers an exceptional revolution in designing the sensors by increasing the surface area and conductivity. Recently, Masteri Farahani et al. have incorporated sites of MIPs with GQDs to improve the sensitivity of MAMP recognition (Fig. 2). By increasing MAMP in this system, the intensity of the FL signal was quenched, while in the presence of other narcotic compounds, no significant change was achieved. This approach provided a selective fluorimetric MAMP assay with a LOD of 12.12 nM (Masteri-Farahani et al., 2020).

Under a similar strategy, $\mathrm{SiO}_{2}$ was synthesized through a sol-gel polymerization route and then the carbon QDs (CQDs) was immobilized on this. The structured mesoporous MIP ( $\left.\mathrm{SiO}_{2} @ \mathrm{QDs} @ \mathrm{~ms}-\mathrm{MIPs}\right)$ was used as a fluorescent probe. The presence of MAMP led to a fluorimetric turn-on system and the MAMP was detected under a linear range of $5-250 \mu \mathrm{M}$ with a LOD of $1.6 \mu \mathrm{M}$ (Fig. 3) (Mandani et al., 2020).

As the bioreceptors that can defeat some limitations of the antibodies, aptamers have been utilized in fluorometric methods in the MAMP measurement. The aptamer is a single synthetic strand of RNA or DNA with exceptional properties presenting high specificity regarding the targets (Roushani and Shahdost-fard 2018; Shahdost-fard and Roushani 2016; Tan et al., 2013). In the last two decades, aptamer-based sensors (aptasensors) have been applied in various applications (Nutiu and Li 2005; Roushani et al., 2020; Shahdost-fard and Roushani 2019, 2020; Yan et al., 2010). Taking advantage of the aptamers, the first fluorometric aptasensor for MAMP detection has been introduced by Saberi et al., in 2018. They applied the cobalt oxyhydroxide $(\mathrm{CoOOH})$ nanosheets as the acceptor and carbon dots (CDs) as the donor (Fig. 4). The interaction of aptamer functionalized with the CDs (aptamer-CDs) with the $\mathrm{CoOOH}$ nanosheets led to a quench of the CDs' fluorescence intensity. By incubating the MAMP to the system and the aptamer configuration change for capturing the MAMP, the aptamer was released from the $\mathrm{CoOOH}$ nanosheets and the FL signal increased. This off-on system detected the MAMP in a linear range of 5-156 nM with a LOD of $1 \mathrm{nM}$ (Saberi et al., 2018).

\subsection{Colorimetric sensors}

Colorimetric detection is a highly regarded method due to its simplicity, low cost and label-freeness. The colorimetric analysis is based on a chemical compound in a solution using a color reagent to detect the target with the naked eye or a portable spectrometer (Kim et al., 2010; Xiao et al., 2015). In recent years nanomaterials make significant progress in colorimetric sensors. Among various nanostructures, metal NPs (MNPs) are the perfect candidate for colorimetric assay because of size-/distance-dependent optical properties. Since a change in inter-particle plasmon coupling leads to a shift of surface plasmon band, the color of colloidal MNPs is sensitive to its aggregation/dispersion. This behavior can be regarded as a useful indicator in the colorimetric assay (Petryayeva and Krull 2011; Zhang et al., 2008). The literature survey reveals that NPs has a crucial role in designing the colorimetric sensor for MAMP analysis. In a study based on the polymer coupled with the NPs, Argente-García et al. synthesized a composite containing 1,2naphthoquinone-4-sulfonate (NQS) and poly dimethylsiloxane/ tetraethylorthosilicate $/ \mathrm{SiO}_{2} \mathrm{NPs}$ (PDMS-TEOS-SiO ${ }_{2} \mathrm{NPs}$ ) for analysis 


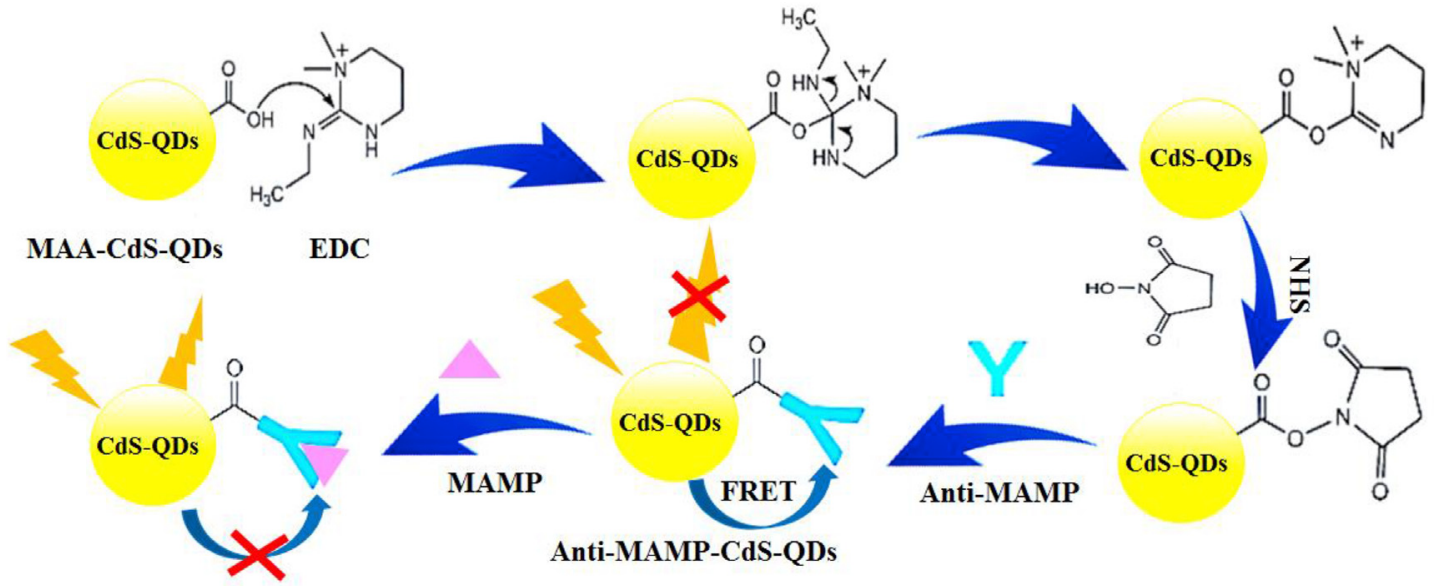

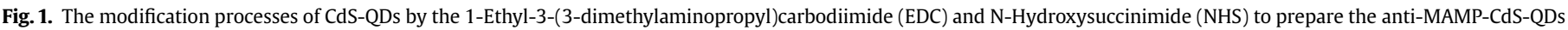
for the MAMP measurement under the FRET mechanism. Reproduced with copyright permission of Springer (Masteri-Farahani and Mosleh 2019).

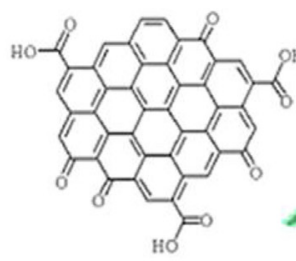

GQDs

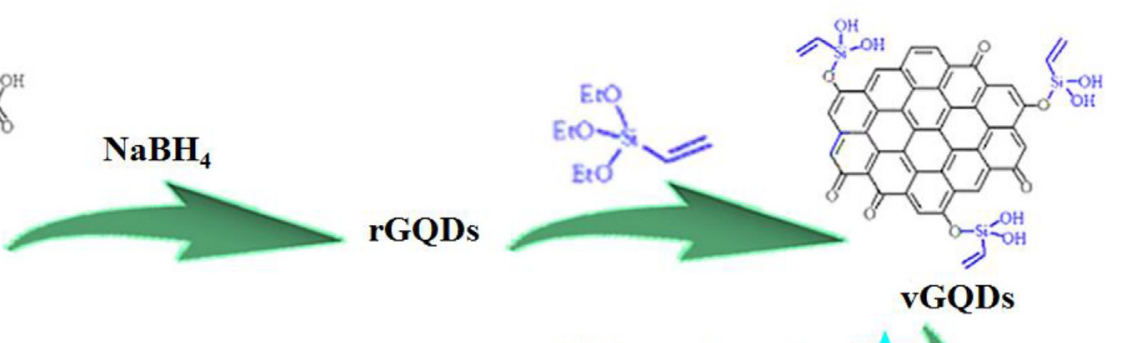

Methamphetamine

Methacrylic acid (MAA)

Ethylene glycol dimethacrylate (EGDMA)

Azobisisobutyronitrile (AIBN)
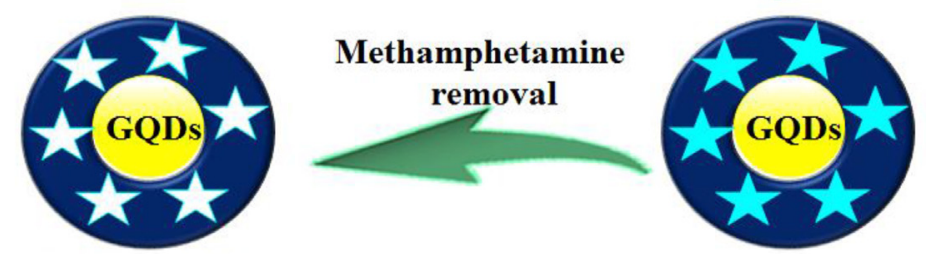

GQDs@MIP

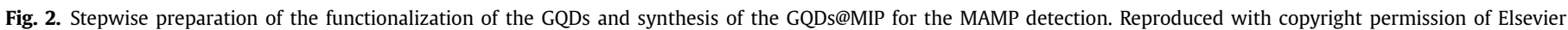
(Masteri-Farahani et al., 2020).

of the drugs from the amphetamine (AMP) family. The drugs include MAMP, 3,4-methylenedioxymethamphetamine (MDMA) and 3,4-methylenedioxyamphetamine (MDA) derivatized inside the composite to embed a colored product in the investigation of the diffuse reflectance or the color intensity. The fabricated kit distinguished the targets in the street samples with a LOD range of 6.46-16.16 mM (Argente-García et al., 2016).

The first colorimetric aptasensor for MAMP detection has been introduced based on the utilization of gold NPs (AuNPs) by Yarbakht's group in 2015. Accordingly, the aptamer structure changed in the absence or presence of the MAMP and the AuNPs was discolored based on the aggregation/dispersion process. This fast visual diagnosis method detected the MAMP in a micromolar range within a few minutes (Yarbakht and Nikkhah 2016).
In another strategy based on the aptasensing approach coupled with NPs, the MAMP was measured by a salt-induced aggregation of unmodified AuNPs. Shi et al. showed the aptamers immobilized to the AuNPs surface have a resistance to the NaCl-induced aggregation. In contrast, in the presence of the MAMP, the aptamer bound to MAMP exhibited a rapid salt-induced aggregation. This aggregation process depends on MAMP, led to a solution color change from red to blue. The feasibility of the aptasensor was validated by visually measuring human urine containing MAMP under a range of $2-10 \mu \mathrm{M}$ with a LOD of $820 \mathrm{nM}$ (Shi et al., 2015).

Combining the aptasensing approach with the NPs and magnetic beads (MBs) provided a sensitive method for the simultaneous analysis of cocaine and MAMP. In this strategy, a specific single-stranded DNA (ssDNA) sequence coated on Au@Ag core- 


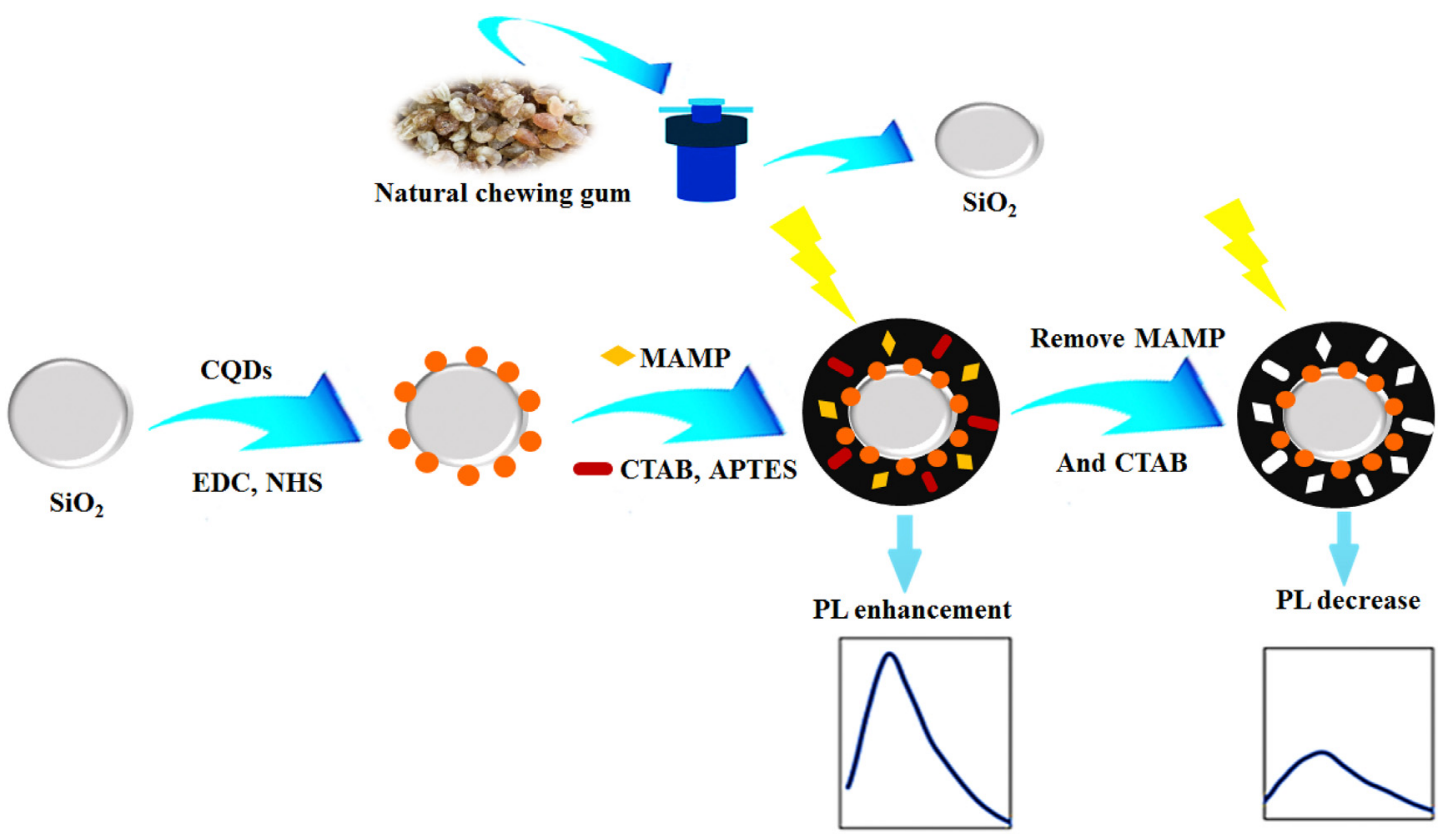

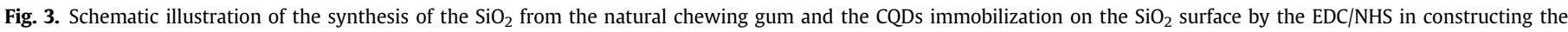
$\mathrm{SiO}_{2} @ Q D s @ m s-M I P s$ for the MAMP sensing. Reproduced with copyright permission of Elsevier (Mandani et al., 2020).

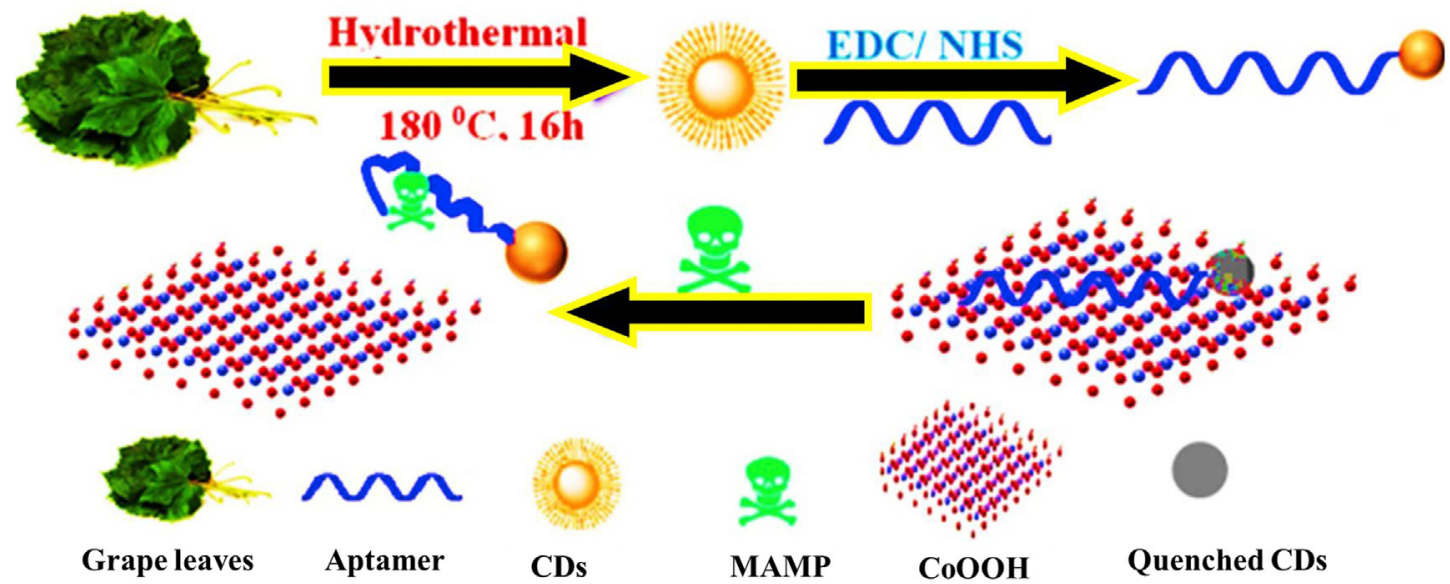

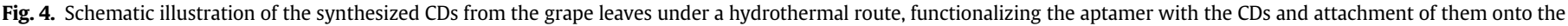
$(\mathrm{CoOOH})$ nanosheets in the MAMP detection. Reproduced with copyright permission of Springer (Saberi et al., 2018).

shell NPs as a reporter probe (RP), a capture probe (CP) conjugated with magnetic beads (MBs), and a target-binding aptamer was used (Fig. 5). The free binding aptamer hybridized with both of the RP and $\mathrm{CP}$ probes in the absence of the targets and no significant color change was detectable by aggregation of the NPs. While introducing the target, a competition occurred for the aptamer to capture the target and the formed target/aptamer complex led to disaggregate the NPs and emit a distinct color. This color change was linearly proportional to different MAMP concentrations in a linear range of $0.5-200 \mathrm{nM}$ with a low LOD of $0.1 \mathrm{nM}$ (Mao et al., 2017).

\subsection{Chemiluminometric sensors}

During the fast multi-step oxidation reactions, a luminescence emission creates the chemiluminescence $(\mathrm{CL})$ process (Zagatto et al., 2011). The CL-based strategy differs from the colorimetric and fluorimetric approaches because of the minimized background interferences arising from excitation light sources and emission filters (Wang et al., 2019). However, this method presents unique advantages but, up to now, only a CL sensor has been reported for MAMP detection. In 2017, Hassanzade's group introduced a MAMP sensor based on both FL and CL methods by utilizing L-cysteine capped CdS QDs, rhodamine B (RhoB) and cetyltrimethylammonium bromide (CTAB). The strategy is based on the fact that the CdS QDs has a promoting effect on the CL reaction of RhoB-CTAB-KMnO ${ }_{4}$ such that adding MAMP leads to a considerable alteration in the FL and $\mathrm{CL}$ emission intensities of the CdS QDs (Fig. 6). Accordingly, the FL emission of QDs was linearly quenched in the presence of $47.97-2.85 \times 10^{3} \mathrm{nM}$ of the MAMP. In contrast, the CL emission enhanced with a linear range of $1.78-891.39 \mathrm{nM}$ in a shorter assay time. The LOD of the CL method was calculated to be $0.613 \mathrm{nM}$, which was over 20 times better than the applied FL method (Hassanzadeh et al., 2017). 


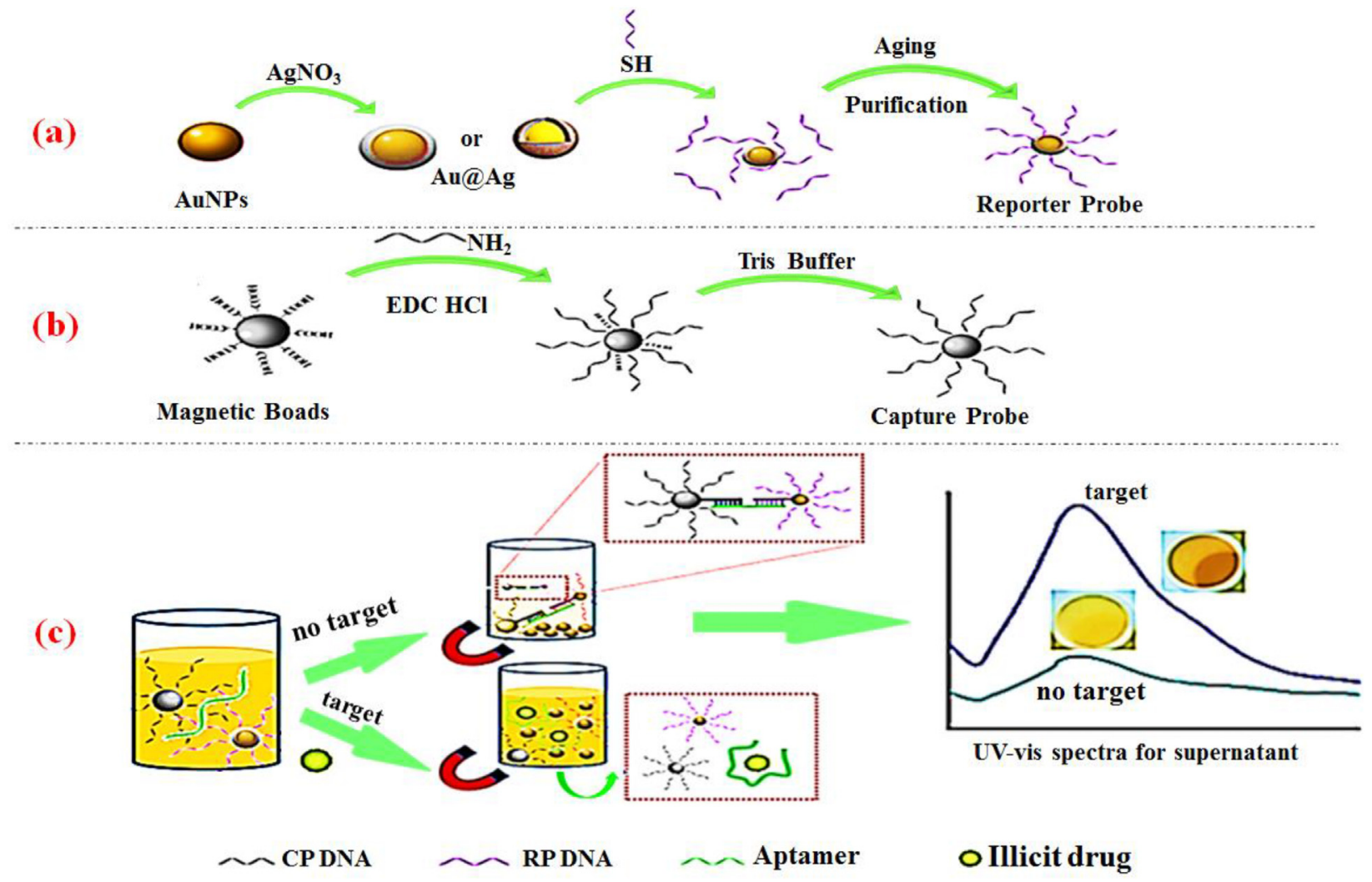

Fig. 5. The preparation of (a) the reported probe, (b) capture probe and (c) the colorimetric aptasensing in the MAMP detection. Reproduced with copyright permission of Elsevier (Mao et al., 2017).

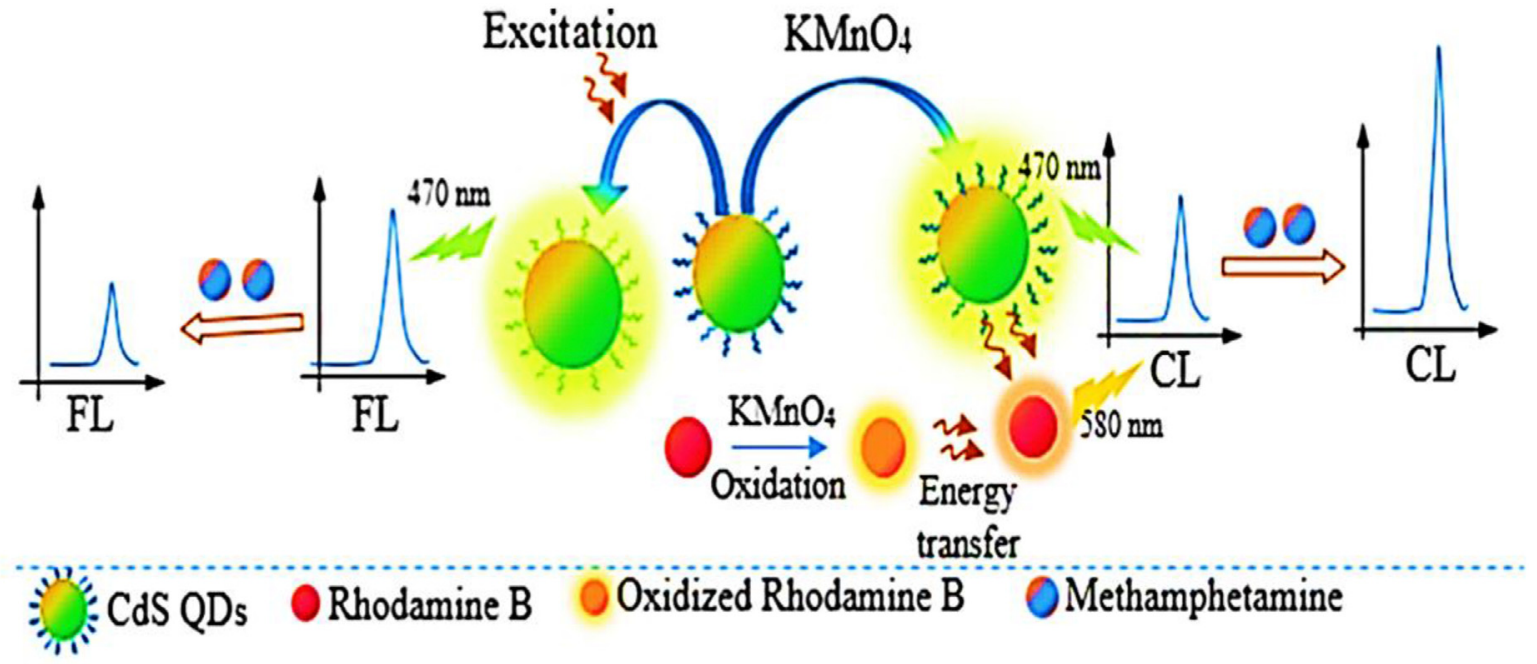

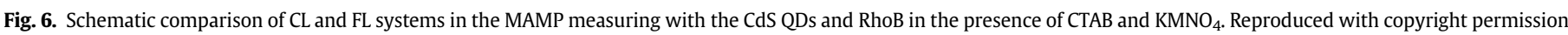
of Elsevier (Hassanzadeh et al., 2017).

\subsection{Surface plasmon resonometric (SPR) sensors}

The surface plasmon resonance (SPR) is an electromagnetic phenomenon created from light interaction with free electrons at a metal-dielectric interface (Piliarik et al., 2007). Accordingly, a refractive index change is achieved by adsorption of target molecules on the specific ligands immobilized on the surface. The SPR sensors benefit from the produced excitation of surface plasmon for optosensing purposes during a short time analysis (Homola 2008; Miyazaki et al., 2017; Ragavan et al., 2013). This approach has been rendered significant consideration in MAMP measuring.

The first SPR immunosensor in MAMP detection has been reported by Sakai et al., in 1999 based on an antigen-antibody interaction. A conjugate of MAMP with bovine serum albumin (BSA) as an antigen (MAMP-BSA) was immobilized on an Au thin film. The related incident angle changed by adding the MAMP 
(a)

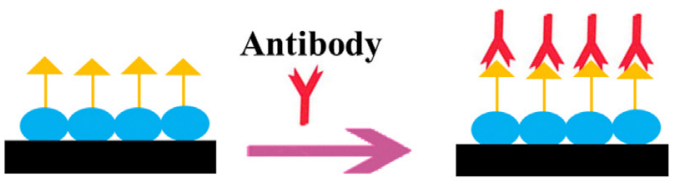

(b)
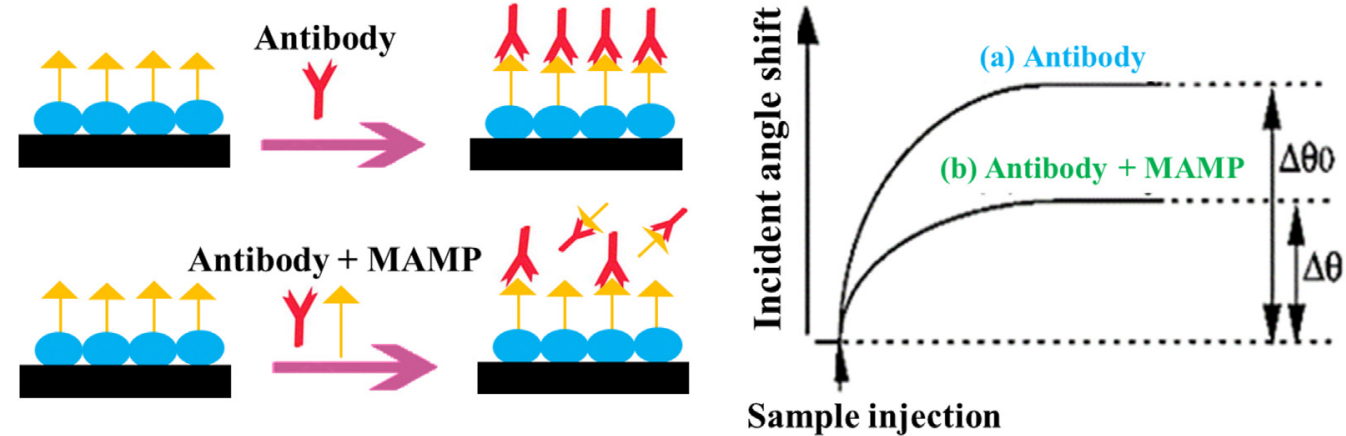

MAMP

Antibody

MAMP

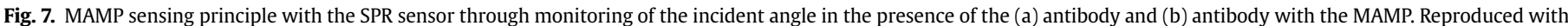
copyright permission of Elsevier (Sakai et al., 1999).

(Fig. 7). Increasing the MAMP to the antibody solution was proportionally decreased the angle in a range of $0.713-7.13 \times 10^{3} \mathrm{nM}$ because of the inhibition effect of MAMP (Sakai et al., 1999).

Based on a similar sensing mechanism, Cao et al. generated another immunosensor in MAMP detection. The serum sample containing MAMP was mixed with the antibody. The concentration increasing of the target bound to the MAMP-BSA conjugate decreased the obtained signal under the same strategy of Sakai's group (Fig. 8). A linear range of $4.92-3.56 \times 10^{3} \mathrm{nM}$ for MAMP was obtained by applying a four-parameter logistic model. The specificity of the immunosensor was confirmed by testing some illicit drugs, including pseudoephedrine, ketamine, morphine and nimetazepam. They indicated the developed immunoassay results were matchable with the standard GC-MS method (Cao et al., 2018).

In another similar study, Chang et al. have reported a fiber optic particle plasmon resonance (FOPPR) immunosensor based on a competitive inhibition in the MAMP determination. They embedded BSA-MAMP conjugate on the fiber core surface modified with the AuNPs. Based on an indirect competitive reaction between the free MAMP in the solution and MAMP bound to the conjugate surface, transmitted light intensity change through the sensor fiber monitored. The MAMP was analyzed in a broad concentration range of $7.13-7.13 \times 10^{3} \mathrm{nM}$ with a LOD of $1.140 \mathrm{nM}$. The FOPPR immunosensor could diagnose MAMP in some diluted human urine samples without nonspecific adsorption interference (Chang et al., 2020).

\subsection{Surface-enhanced Raman scattering (SERS) sensors}

Surface-enhanced Raman scattering (SERS) is a surface-sensitive phenomenon that enormously enhances the resulted Raman scattering signals through absorbed molecules on metal surfaces or nanomaterials. In this technique, the enhancement of the electromagnetic-field of plasmonic substrates with the vibrational Raman spectroscopy chemical specificity is applied to measure the analyte (Wang et al., 2017; Zheng and He 2014). The SERS-based sensors have presented potential in illegal drug detection because of their non-invasive probing, minimal sample preparation,

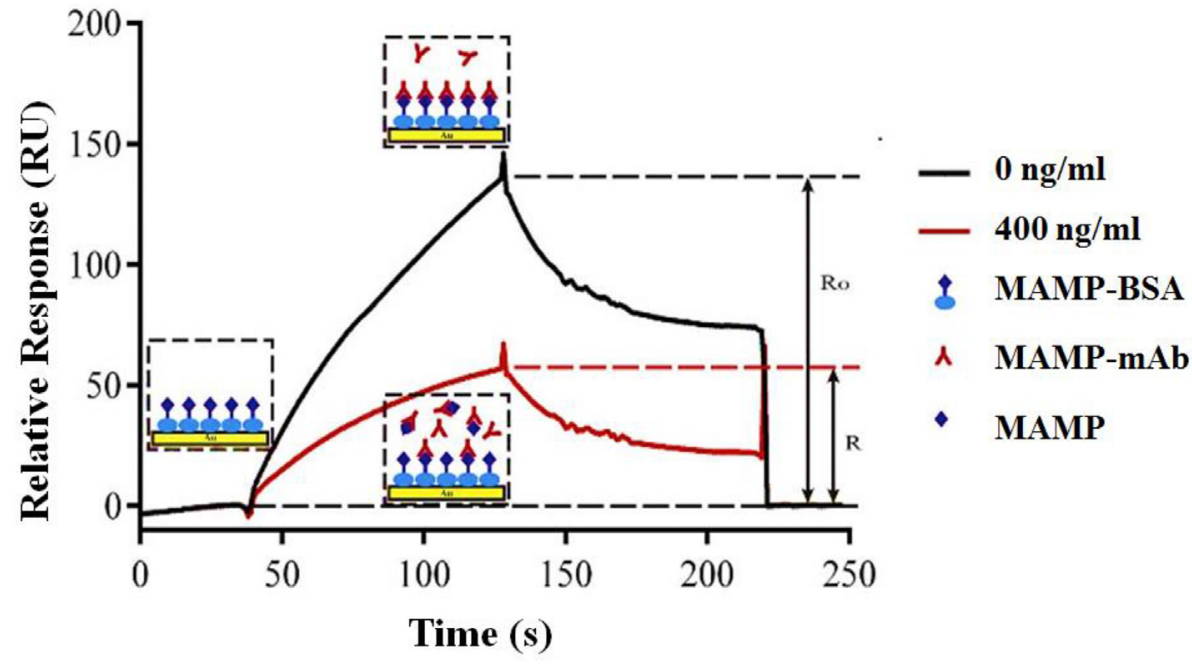

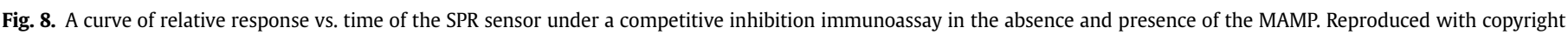
permission of Elsevier (Cao et al., 2018). 
compatibility with an aqueous solution, and label-free monitoring for a specific analyte in complex matrices (Yu et al., 2019).

For the first time, SULK et al., in 1999 developed a SERS method to detect the AMP and MAMP. A coupling reaction was done by 2mercaptonicotinic acid (2-MNA) and dicyclohexylcarbodiimide (DCC) to form two amide compounds of AMP and MAMP. The compounds were detected based on comparing the intensity of the resulted Raman bands relative to a Raman band of the internal standard by a LOD value of $140 \mu \mathrm{M}$ (AMP) and $114 \mu \mathrm{M}$ (MAMP) (Sulk et al., 1999).

In another study, Nuntawong et al. by applying a nanotechnology-based strategy for the first time in the SERS sensors, offered the vertically-aligned silver nanorods-based SERS chips to measure MAMP and AMP in some human urine samples. The SERS substrates were achieved by silver nanorods synthesized based on the magnetron sputtering technique. The analysis was done in the acidulation-treated urine samples and a LOD value of $335.03 \mathrm{nM}$ was obtained (Nuntawong et al., 2017).

Mao's group in 2018 took advantage of the aptamer benefits for the first time in the SERS sensors to prepare a selective substrate to detect MAMP (Fig. 9). The applied strategy was based on 4mercaptobenzoic acid (4-MBA) labeled core-shell Au@Ag nanoparticles and aptamer. The signal intensity of 4-MBA as the Raman reporter significantly intensified due to the gold@silver (Au@Ag) aggregation and hot spots SERS formation. The sensor detected MAMP under a logarithm relationship between the SERS signal and MAMP concentration with a linear range of 3.35-268.03 nM and LOD of $1.072 \mathrm{nM}$. The results were confirmed by the MAMP detection through HPLC-MS/MS in the human urine sample (Mao et al., 2018).

Salemmilani et al. reported a dielectrophoresis-induced microfluidic SERS device with a high capability in the MAMP analysis in some saliva samples for 2 min. Accordingly, iodide-modified AgNPs was trapped and released by electrodes integrated into a microfluidic channel. Chemometrics studies based on the principal component analysis (PCA) were done to classify the MAMP-positive samples from the negative control samples. The sensor presented reuse capability multiple times because the passivation of the electrodes and flow channels decrease the microchannel fouling by the AgNPs (Salemmilani et al., 2018).

In another study, Au nanorods (AuNRs) arrays were fabricated based on a simple and maskless nanofabrication process of Oblique angle deposition (OAD) to provide uniform substrates for SERS studies. Based on the finite-difference time-domain (FDTD) investigation, the maximum electromechanical (EM) field intensity was calculated by length and the tilting angle of AuNRs. Using the probe molecule of 1,2-bis (4-pyridyl) ethylene (BPE) in MAMP detection, a LOD value of $10^{-11} \mathrm{M}$ was detected in human urine (Li et al., 2019).

A SERS-active microcavity as a MAMP sensor was developed by Fang et al., in 2020. This device was fabricated based on microcavities distributed on a glass array and compact self-assembly nanopeanuts on the inside surface. Crystal violet acetate (CVa) as the internal standard label was anchored inside the middle gap of the CSNPs on the surface of microcavity arrays. This device was equipped with a manual monolithic column syringe needle to detect MAMP spread on the microcavity array bottom area. The sensor was able to on-site detect $67 \mathrm{nM}$ in the urine and serum samples (Fang et al., 2020).

Hong et al. reported optoplasmonic hybrid nanomaterials as the SERS substrate for the sensitive detection of MAMP. Accordingly, a MAMP-carrying dielectric layer was attached to AuNPs monolayer to enhance the electric field localization intensity and provide more MAMP in the electromagnetic "hot" surface. The resulted sensor was detected MAMP in saliva and urine at nanomolar concentrations (Hong et al., 2020).

In another study based on the MAMP aptamer, an aligner mediated cleavage (AMC) of DNA using a MAMP aptamer was introduced by Mao et al., in 2021. In this study, by sequence-specific cleavage of aptamer linked by AuNPs (aptamer-AuNPs), hybridization of the cleavage aptamer-AuNPs with complementary DNA (cDNA)-AuNPs led to aggregate the AuNPs and concomitant plasmonic coupling effect. Accordingly, the interparticle distance of the AuNPs was decreased and the electric field enhancement factor was enhanced by reducing the base number of aptamer-AuNPs. So, rhodamine 6G (R6G) adsorbed on AuNPs surface produced a strong Raman signal under the laser irradiation. The sensor detected MAMP under a linear range of $10 \mathrm{pM}$ to $10 \mathrm{nM}$ with a LOD of $7 \mathrm{pM}$ (Mao et al., 2021).

Although the conventional SERS method on the portable Raman spectrometer has still challenges because of the spectra process with manual intervention and low sensitive Raman signal, the dynamic SERS (DSERS) method presented some benefits in the

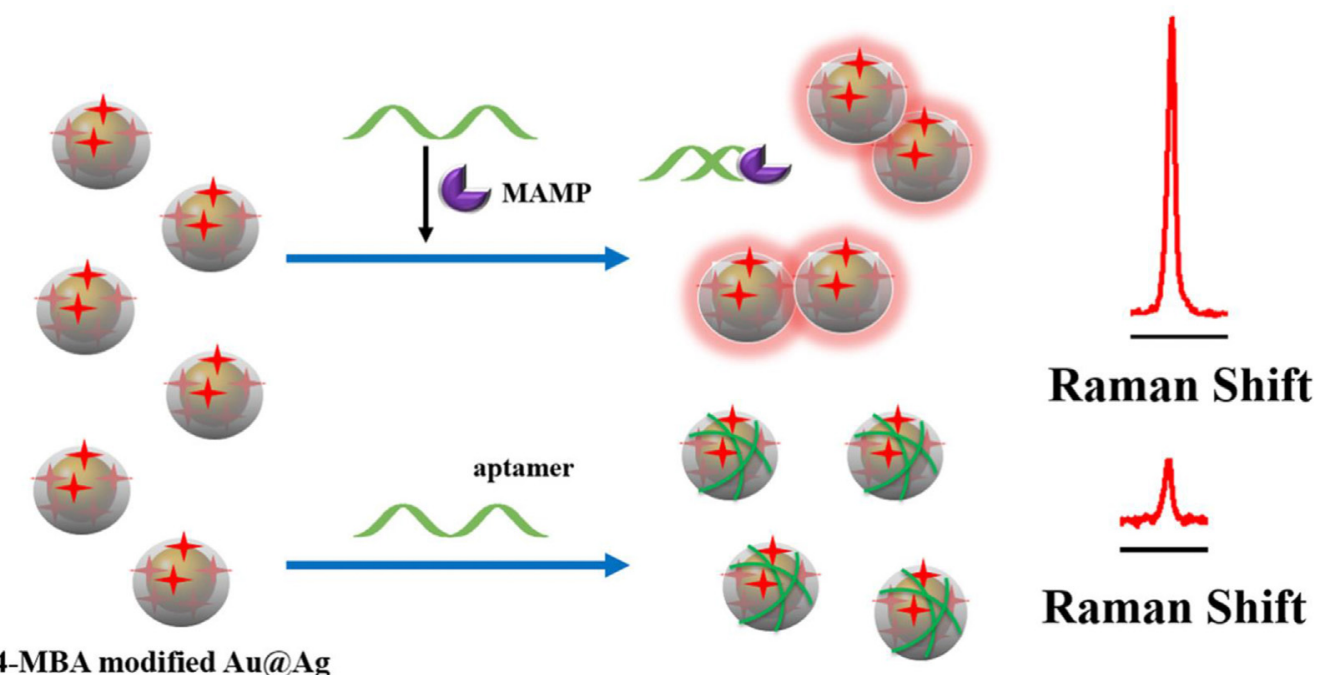

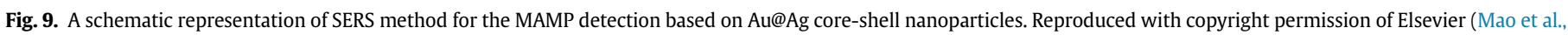
2018). 
better analyte detection. This method removes normal Raman and instrumental interferences and does site-selective spectroscopy of adsorbate populations on active particles better than the SERS method. Accordingly, Dong et al. developed a DSERS-based sensor by utilization a portable Raman spectrometer on AuNRs to detect 3, 4-methylenedioxy methamphetamine (MDMA) and MAMP in human urine. Data obtained from the analysis of urine in the absence and presence of analyte were collected based on the generation of high reproducible SERS signals by the AuNRs. A support vector machine (SVM) model as a classification algorithm was made. Both the MDMA and MAMP were detected in the addicts' urine. The analysis was directly done with no need for the corresponding SERS spectra by $2 \mu \mathrm{L}$ sample volume during 2 min (Dong et al., 2015).

Under the same strategy, Weng et al. presented a DSERS method based on the mPEG-SH coated AuNRs and discriminate MAMP and MDMA in human urine samples by the chemometric studies. A recognition model was made based on the selection of spectra at the critical state by K-means and proposing random forest (RF) with feature selection and PCA. The analytes were discriminated by the classification model and identification accuracy was investigated. Using the chemometric studies in this method helps to a better detection process during some seconds (Weng et al., 2018).

There are some other optical sensors in the MAMP detection in literature from which the most important ones are summarized in Table 1.

\section{Electrochemical sensors in MAMP detection}

The electrochemical sensors are devices that couple a material to an electrode transducer. These electrodevices present some advantages compared to the optosensors. The performance of an electrochemical sensor depends on how the receptor immobilization on the electrode surface to prepare an efficient interface sensing. Since the stabilization of the diagnostic agents such as MIP, antibody, protein, aptamer, and enzyme on the electrode surface is of great importance in sensing devices, applying a suitable sublayer is pivotal in the electrode modification process. Many electrochemical sensors based on several techniques have been introduced in the selective and sensitive detection of various analytes (Freire et al., 2003; Karim-Nezhad et al., 2016; Razmi et al., 2019; Rosy and Yadav 2014; Roushani et al., 2019; Shahdost-fard and Roushani 2017). The electrochemical MAMP sensors are classified based on the applied techniques as voltammetric, potentiometric, impedimetric, amperometric and electrochemiluminometric sensors (Scheme 4)(Karimi-Maleh et al., 2020). The following subsections summarize the electrochemical sensors for MAMP detection.

\subsection{Voltammetric sensors}

Voltammetric strategy shows excellent sensitivity and selectivity due to the analyte can be easily recognized by its voltammetric peak (Ganjali et al., 2017; Moshirian-Farahi et al., 2020; Taghdisi et al., 2016; Tajik et al., 2018).

\subsubsection{Cyclic voltammetry (CV)-based sensors}

The cyclic voltammetry (CV) technique is a common voltammetry technique in electrochemical studies, which works based on redox reactions. Some kinetic parameters such as the active surface area and the surface coverage of the modified electrode, current density changing, and transfer coefficient of reaction can be evaluated by this technique (Baghbamidi et al., 2016; Foroughi et al., 2014; Rezaei and Irannejad 2019; Tajik and Beitollahi 2019). Due to this technique is not so sensitive, rather than the other electrochemical techniques in the target detection, up to now, only one $\mathrm{CV}$-based sensor has been reported for the MAMP measurement. Kohzadi et al., in 2016 have used a nanocomposite containing chitosan and AuNPs as a sub-layer to introduce the first MAMP aptasensor in the CV-based sensor class. This first application of nanotechnology in the voltammetric MAMP sensor improves the

Table 1

The optical sensors for MAMP detection.

\begin{tabular}{|c|c|c|c|c|c|}
\hline Method & Interface & Linear Range $(\mu \mathrm{M})$ & LOD (nM) & Matrix & Ref. \\
\hline Fluorimetric & Polyfluorene- $\mathrm{NH}_{2}$ & 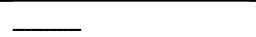 & 178.58 & & Cheng et al. (2011) \\
\hline Fluorimetric & FBT \& PFT & $\longrightarrow$ & $1.35 \times 10^{4} \& 4.56 \times 10^{4}$ & & Fu et al. (2013) \\
\hline Fluorimetric & Benzothiadiazole & & $1.28 \times 10^{3}$ & & Wen et al. (2012) \\
\hline Fluorimetric & NTS@SiO ${ }_{2} \mathrm{NPs}$ & $6.7-270$ & 1.0 & & Rouhani and Haghgoo (2015) \\
\hline Fluorimetric & Anti-MAMP-CdS QDs & $0.14-17.82$ & 42.78 & & Masteri-Farahani and Mosleh (2019) \\
\hline Fluorimetric & GQDs@MIP & $5-50$ & 42.78 & & Masteri-Farahani et al. (2020) \\
\hline Fluorimetric & $\mathrm{SiO}_{2} @ \mathrm{QDs} @ \mathrm{~ms}-\mathrm{MIPs}$ & $5-250$ & $1.6 \times 10^{3}$ & Urine, Blood & Mandani et al. (2020) \\
\hline Fluorimetric & Aptamer/CoOOH-CDs & $5 \times 10^{3}-1.56 \times 10^{5}$ & 1 & Plasma & Saberi et al. (2018) \\
\hline Fluorimetric & GQDs & 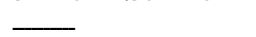 & $10.55 \times 10^{9}$ & & Masteri-Farahani and Askari (2019) \\
\hline Fluorimetric & Polymer prob & 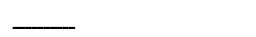 & - & - & Yu et al. (2020) \\
\hline Colorimetric & PDMS-TEOS-SiO ${ }_{2} \mathrm{NPs}$ & & $2.85 \times 10^{7}$ & Street sample & Argente-García et al. (2016) \\
\hline Colorimetric & Aptamer/AuNPs & $5-400$ & & & Yarbakht and Nikkhah (2016) \\
\hline Colorimetric & Salt/Aptamer/AuNPs & $2-10$ & 820 & Urine & Shi et al. (2015) \\
\hline Colorimetric & Aptamer/Au@Ag core-shell & $5 \times 10^{-4}-.200$ & 0.1 & Urine & Mao et al. (2017) \\
\hline Colorimetric & Aptamer/Gquadruplex-hemin DNAzyme & $8 \times 10^{-3}-0.500$ & 0.5 & Urine & Mao et al. (2016) \\
\hline Chemiluminescence & $\mathrm{KMnO}_{4}$-RhoB-CTAB/CdS QDs & $1.7 \times 10^{-3}-0.89$ & 0.613 & Urine & Hassanzadeh et al. (2017) \\
\hline SPR & Anti-MAMP-BSA & $7.13 \times 10^{-4}-7.13$ & & Urine & Sakai et al. (1999) \\
\hline SPR & Anti-MAMP-BSA & $4.92 \times 10^{-3}-3.56$ & 0.92 & Serum & Cao et al. (2018) \\
\hline SPR & AuNPs-BSA-Anti-MAMP & $7.13 \times 10^{-3}-7.13$ & 1.14 & Urine & Chang et al. (2020) \\
\hline SERS & 2-mercaptonicotinic acid & 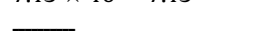 & $1.14 \times 10^{5}$ & Urine & Sulk et al. (1999) \\
\hline SERS & 4-MBA modified Au@Ag & $3.3 \times 10^{-3}-0.26$ & $1.07 \times 10^{-3}$ & Urine & Mao et al. (2018) \\
\hline SERS & AgNRs & - & $3.3 \times 10^{2}$ & Urine & Nuntawong et al. (2017) \\
\hline SERS & Core-shell nanopeanuts & $\bar{\tau}$ & - & Urine, Serum & Fang et al. (2020) \\
\hline SERS & AuNRs & $\bar{\square}$ & $10^{-7}$ & Urine & Li et al. (2019) \\
\hline SERS & MAMP aptamer-Au NPs & $10 \times 10^{-6}$ to $10 \times 10^{3}$ & $7 \times 10^{-3}$ & Serum & Mao et al. (2021) \\
\hline SERS & Iodide-modified AgNPs & - & - & Saliva & Salemmilani et al. (2018) \\
\hline SERS & Optoplasmonic & $\longrightarrow$ & 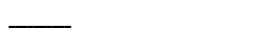 & Saliva, Urine & Hong et al. (2020) \\
\hline D-SERS & AuNRs & $\bar{\square}$ & $\bar{\square}$ & Urine & Dong et al. (2015) \\
\hline D-SERS & mPEG-SH coated AuNRs & {[} & 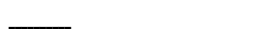 & Urine & Weng et al. (2018) \\
\hline
\end{tabular}




\section{Electrochemical methods for MAMP detection}

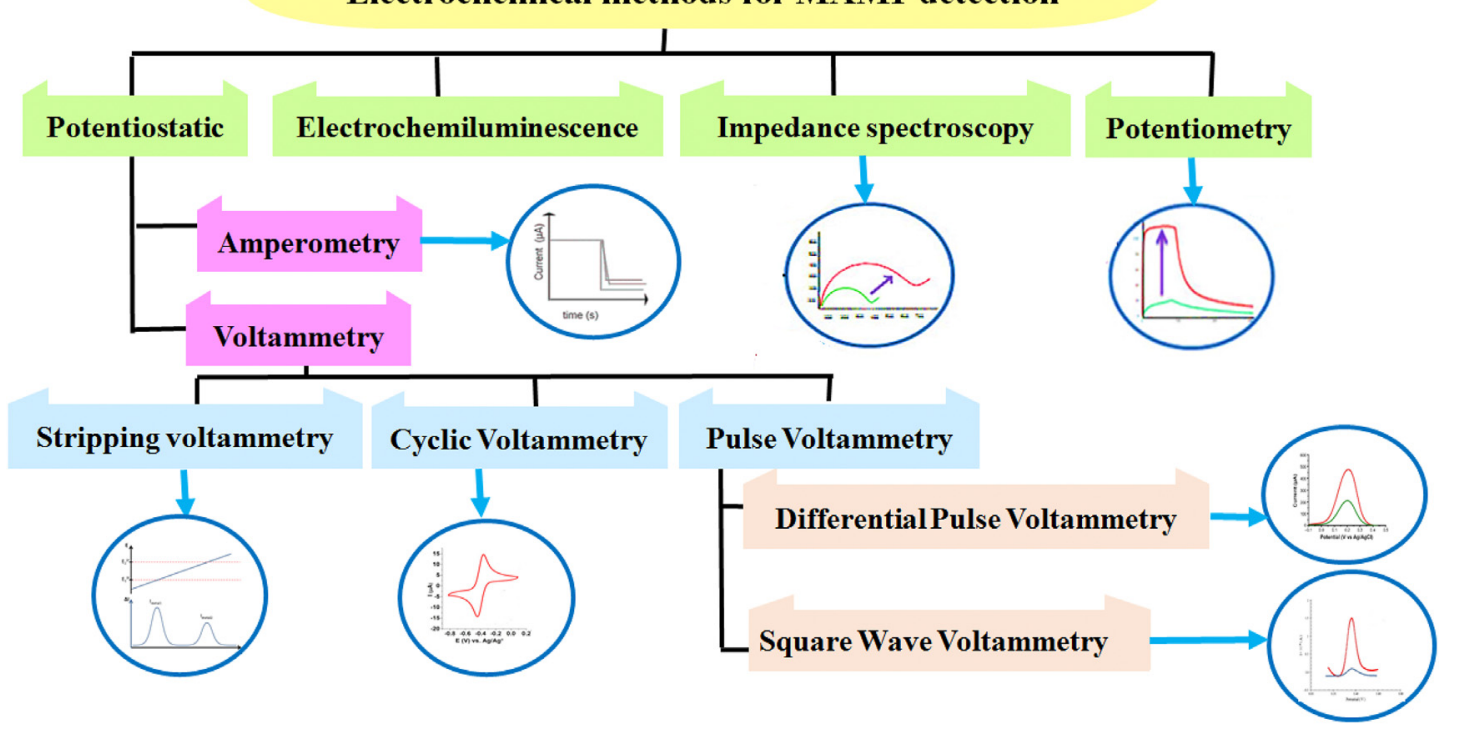

Scheme 4. Classification of various electrochemical methods of the MAMP sensor.

great mechanical stability of chitosan (CHIT) with the AuNPs to embed a suitable platform to capture the thiolated-aptamer sequence on the modified electrode surface (Fig. 10). By incubation of MAMP on the aptasensor surface, the conformation of the aptamer changed to capture the target and a further change in the $\mathrm{CV}$ signal of the ferro/ferricyanide $\left(\left.\left[\mathrm{Fe}(\mathrm{CN})_{6}\right]^{3-}\right|^{4-}\right)$ as a redox probe was achieved. Besides, they used a microcantilever-based sensor to compare the corresponding results. The LOD for MAMP sensing was calculated to be $10 \mathrm{nM}$ and $0.7 \mathrm{nM}$ for the electrode modification and microcantilever approaches, respectively (Kohzadi et al., 2016).

\subsubsection{Differential pulse voltammetry (DPV)-based sensors}

The differential pulse voltammetry (DPV) popular technique presents a more sensitive response rather than the CV technique. This behavior is rooted in the fact that the current is measured at each pulse to minimize the background current, which is useful in trace analysis of the analytes (Bockris 1981).

Lubomir Svorc's group has reported a DPV-based sensor to MAMP measurement by a self-assembled boron-doped diamond electrode (BDDE) as an efficient platform. The BDDE exhibited an irreversible peak related to the MAMP oxidation at $+1.23 \mathrm{~V}$ (vs. Ag/ $\mathrm{AgCl}$ ) in the Britton-Robinson buffer (BRB) solution with $\mathrm{pH}=10 . \mathrm{A}$ two-proton/two-electron mechanism based on the oxidation of secondary amino group in the formation of hydroxylamine in MAMP structure was proposed. The MAMP assay was done based on the DPV technique under a linear range of $0.07-80 \mu \mathrm{M}$ with a LOD of 50 nM. (Švorc et al., 2014).

In another study, Oghli et al. used a pencil graphite electrode (PGE) to fabricate an electrochemical sensor for the MAMP detection. The PGE electrochemical activity enhancement was attributed to the oxygen-containing group increase on the surface (phenolic, carbonyl, and carboxyl groups) or the graphite oxide film formation during electrochemical pretreatment of the PGE in the BRB solution $(\mathrm{pH}=11)$. This issue boosted the signal obtained based on the interacts of the MAMP with the surface (Oghli et al., 2015). The MAMP was quantitatively measured in the blood, urine and seized drug samples by the DPV technique under a dynamic linear range of $0.074-54 \mathrm{mM}$ with a LOD of $50 \mathrm{nM}$ (Fig. 11).

Demir and coworker modified a glassy carbon electrode (GCE) with a fluorescent-labeled polypeptide (EDOT-BTDA-Pala) as a platform to immobilize the MAMP-antibody via cross-linking of amino groups. The GCE/EDOT-BTDA-Pala/antibody as the sensor was applied to analyze MAMP in saliva, urine and serum samples (Fig. 12). Based on the DPV results, the MAMP was distinguished in a dynamic range of 71.31-713.11 $\mu \mathrm{M}$ and a LOD of 93.20 $\mu \mathrm{M}$ (Demir et al., 2016).

\subsubsection{Square wave voltammetry (SWV)-based sensors}

In the square wave voltammetry (SWV) technique, a symmetrical square wave superimposes on a staircase waveform where the forward pulse of the square wave (with a pulse direction same as the scan direction) coincides with the staircase step. Thus, the SWV technique produces a faster signal than the DPV technique, which can be favorable for routine quantitative analyses (Lovrić 2010; Osteryoung and Osteryoung 1985).

Accordingly, Garrido et al. developed a voltammetric sensor in distinguishing four illicit drugs, including AMP, MAMP, MDA and MDMA. The electrochemical study of an unmodified GCE for the drugs in different buffer solutions provided some information about the analyte content and amount in the ecstasy tablets (Garrido et al., 2010). The oxidation mechanism of these electroactive analytes is related to the oxidation of the aromatic nucleus and the secondary amine group present in the molecules. A LOD of $1.2 \mu \mathrm{M}$ based on the SWV studies for the MDMA detection was reported.

The first application of a screen-printed carbon electrode (SPCE) in MAMP analysis has been reported in the SWV-based sensor. This type of polished-free electrodes, by offering some advantages such as high surface area, microliter solution volume and low cost, are superior to the traditional electrodes such as GCE and PGE in sensing applications (Taleat et al., 2014). Accordingly, Bartlett's group used the SPCE as the working electrode and an intermediate of N,N'-(1,4-phenylene)-dibenzenesulfonamide to measure MAMP. The oxidized mediator reacted with MAMP on the SPCE surface and an electrochemical reduction process was done. By applying a double square wave reduction technique for the MAMP sensing in the undiluted saliva, a LOD of $1.29 \mu \mathrm{M}$ during $55 \mathrm{~s}$ was obtained (Bartlett et al., 2016).

Carbon nanotubes (CNTs) as nanomaterials with fantastic 


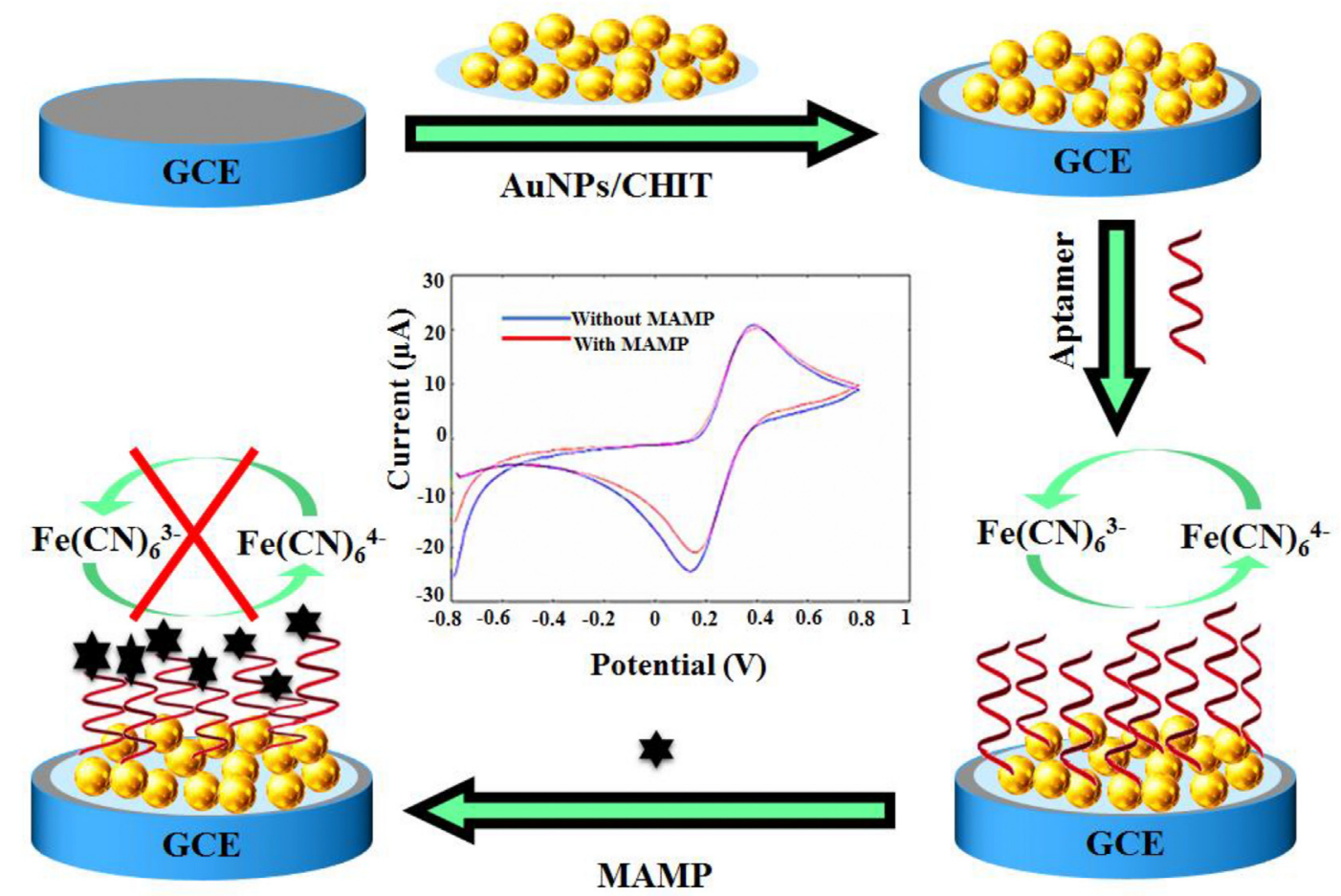

Fig. 10. Schematic illustration of a GCE modified with the AuNPs/CHIT and aptamer sequence in MAMP aptasensing through underlying the ferro/ferricyanide redox probe. (Kohzadi et al., 2016).

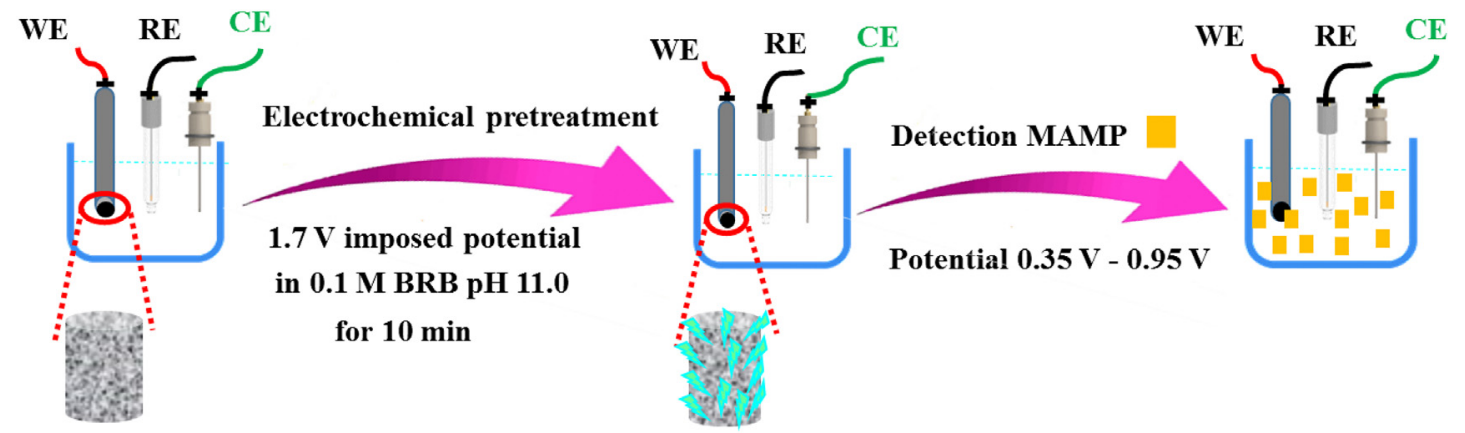

Fig. 11. Schematic electrochemical pretreatment of the PGE in the detection of MAMP and the corresponding DPV curve.

properties including high electron transfer, a great surface to volume ratio, excellent flexibility, unique chemical and thermal durability are a favorable choice in the fabrication electrochemical sensors (Karim-Nezhad et al., 2018; Razmi et al., 2013; Vashist et al., 2011). Accordingly, Alizadeh et al. introduced the first application of nanotechnology in the MAMP SWV-based sensor fabrication by utilizing multi-walled CNTs (MWCNTs). A carbon paste electrode (CPE) was modified with the nanocomposite of MIP/MWCNTs to provide the MIP/MWCNTs/CPE as the electrochemical MAMP sensor (Fig. 13). The well-defined oxidation peak of the MAMP on the modified CPE at $1 \mathrm{~V}$ corresponded to the oxidation of the secondary aliphatic amine in the MAMP structure compared to the lower signal of the unmodified CPE. The fast Fourier transform square wave voltammetry (FFT-SWV) technique with the signal intensity amplifying capability was applied in the MAMP analysis. The sensor measured MAMP under a linear dynamic range of 0.01-100 $\mu \mathrm{M}$ with a LOD of $0.83 \mathrm{nM}$ (Akhoundian et al., 2019).

Haghighi and coworkers reported another application of the MWCNTs in the SWV-based sensor development for MAMP detection. They utilized a GCE modified with the aminefunctionalized MWCNTs and AuNPs linked to the silica-coated magnetite $\left(\mathrm{SiO}_{2} @ \mathrm{Fe}_{3} \mathrm{O}_{4}\right)$ nanoparticles to prepare the $\mathrm{GCE} /$ MWCNT/AuNPs-SH- $\left(\mathrm{CH}_{2}\right)_{3}-\mathrm{Si}-\mathrm{SiO}_{2} @ \mathrm{Fe}_{3} \mathrm{O}_{4}$ as the sensor. The bare GCE surface exhibited a weak irreversible oxidation peak of the MAMP, while the GCE/MWCNT/AuNPs-SH- $\left(\mathrm{CH}_{2}\right)_{3}-\mathrm{Si}-\mathrm{SiO}_{2} @ \mathrm{Fe}_{3} \mathrm{O}_{4}$ indicated a sharp anodic peak under the diffusion-controlled mechanism. The $\mathrm{SiO}_{2} @ \mathrm{Fe}_{3} \mathrm{O}_{4}$ nanoparticles through the outer sphere electron transfer mechanism facilitate the electron transfer between MAMP and amine-functionalized MWCNTs. The sensor detected MAMP based on the SWV signal in three linear ranges from 0.05 to $50 \mu \mathrm{M}$ with a LOD of $16 \mathrm{nM}$ (Haghighi et al.).

Reduced graphene oxide (rGO), as another admirable nanomaterial in electrochemical sensor fabrication, has presented some beneficial properties in MAMP detection. Recently, Anvari et al. prepared a GCE modified with cerium oxide nanoparticles $\left(\mathrm{CeO}_{2} \mathrm{NP}\right)$ decorated on rGO to construct the $\mathrm{CeO}_{2} \mathrm{NP} / \mathrm{rGO} / \mathrm{GCE}$ as the sensor in MAMP screening. The results exhibited a significant current signal and reduced overvoltage toward MAMP on the 


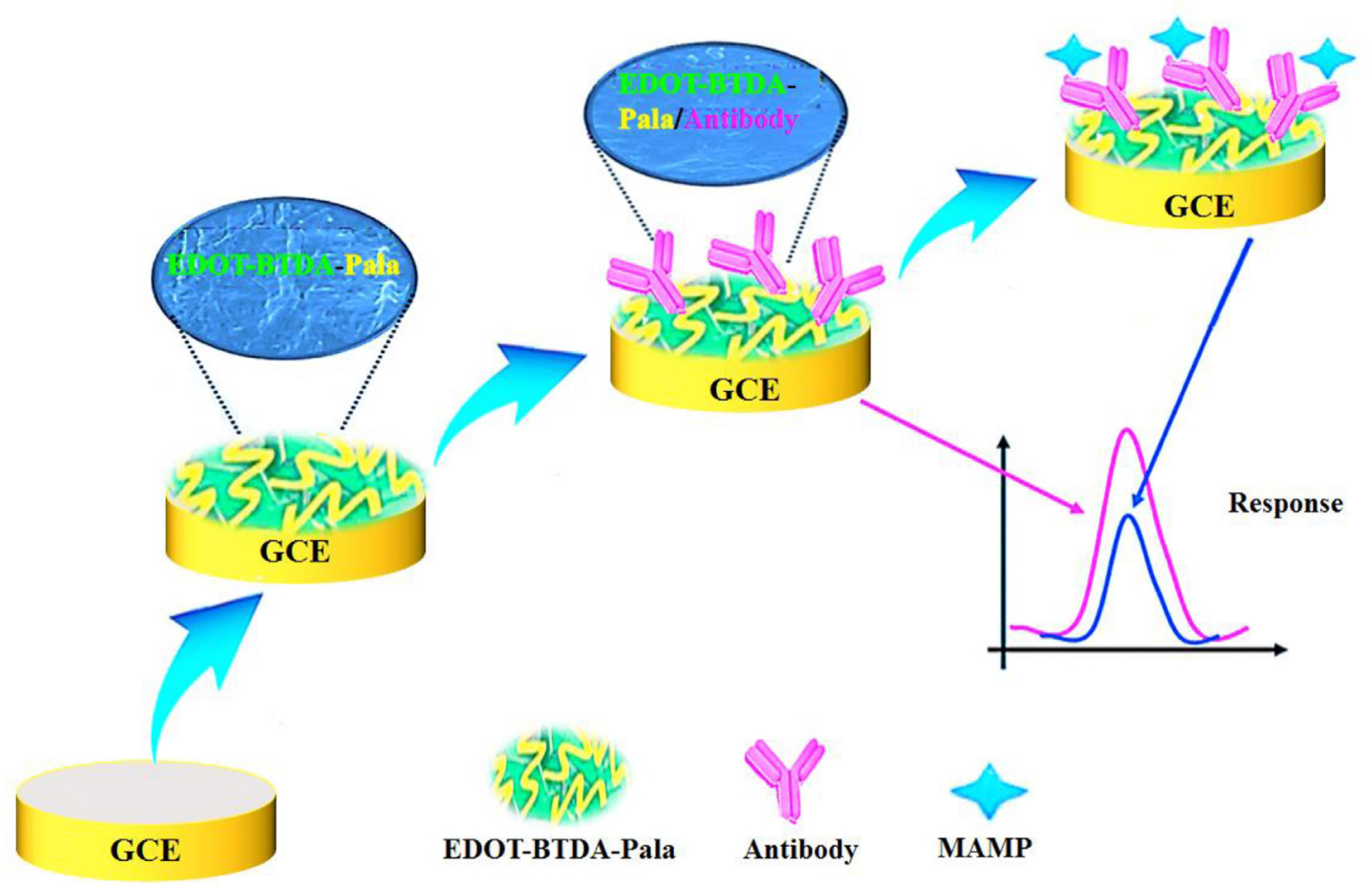

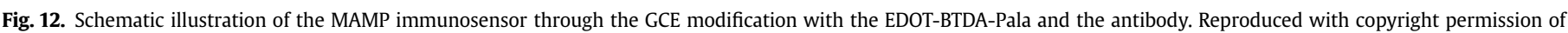
Elsevier (Demir et al., 2016).

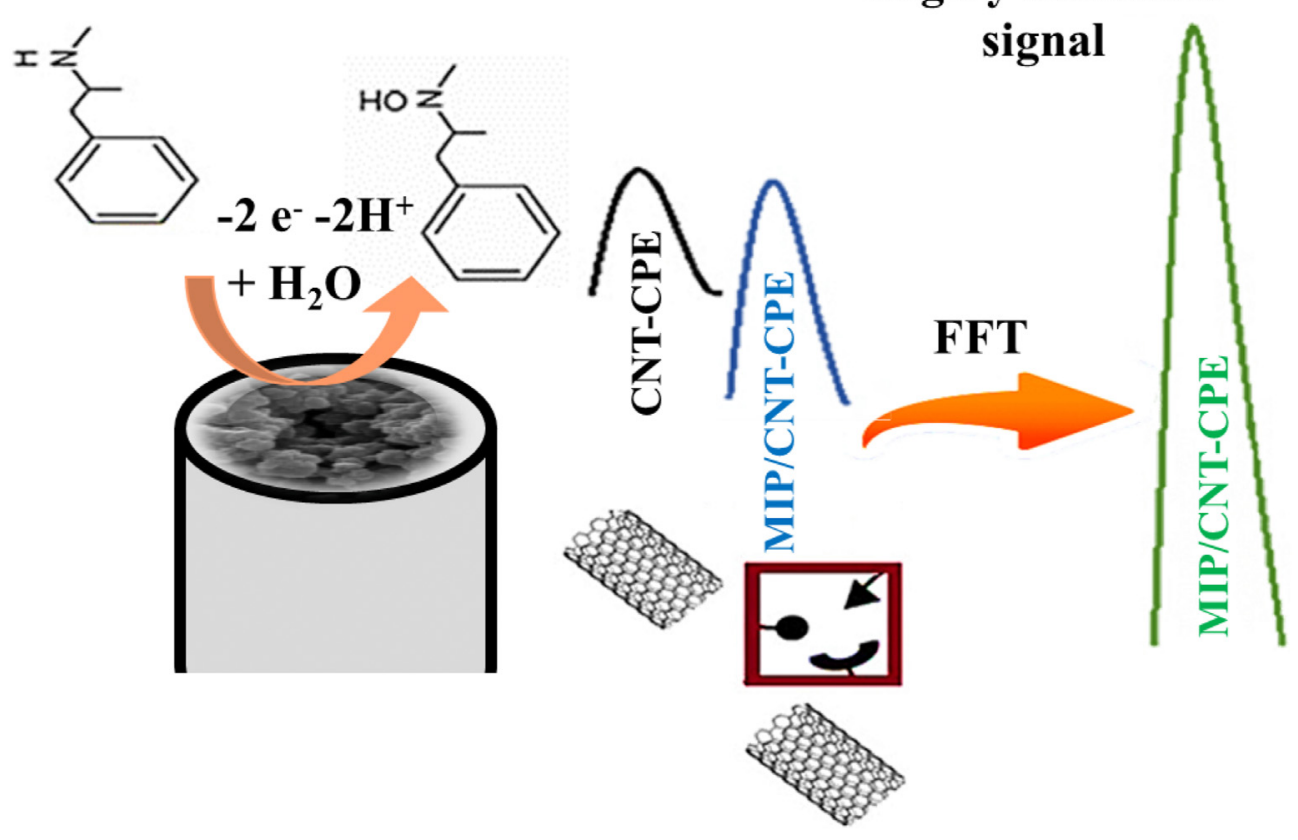

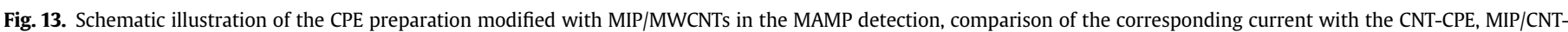
CPE, and improvement of the signal by applying the FFT-SWV technique. Reproduced with copyright permission of Elsevier (Akhoundian et al., 2019).

sensor surface compared to the unmodified GCE. The MAMP was oxidized based on a chemical reaction (EC) mechanism involving two-electrons and two-protons under a linear dynamic range from 25 to $166.6 \mu \mathrm{M}$ and a LOD of $8.7 \mu \mathrm{M}$ (Anvari et al., 2021).

\subsection{Potentiometric sensors}

The potentiometric sensor works on the electrical potential of a modified electrode when there is no current. The utilization of this 
class of the sensor in MAMP detection is only limited to an old study in 1993 by Watanab's group. They prepared a sensitive membrane electrode as a sensing tool to detect MAMP in urine samples. The sensor was fabricated by incorporating sodium tetrakis[3,5-bis(trifluoromethyl)phenyl]borate as the ion exchanger and tricresyl phosphate as the membrane solvent in a membrane matrix of poly(vinyl chloride). The results of the potentiometric studies presented a LOD value of $10 \mu \mathrm{M}$ in the MAMP measurement (Watanabe et al., 1993).

\subsection{Impedimetric sensors}

The studies based on the electrochemical impedance spectroscopy (EIS) technique, known as the impedimetric studies, are very informative in investigating some parameters such as characterization of charge transfer, double-layer constitution, corrosion and kinetic studies (Pérez-Fernández et al., 2020; Rezaei and Irannejad 2019; Roushani and Shahdost-fard 2015). Accordingly, as another class of sensors, the impedimetric sensors have presented useful information in MAMP detection.

Ebrahimi's group in 2012 have introduced the first impedimetric aptasensor in MAMP measuring. They combined two methodologies based on nanotechnology and aptasensing for the first time to screen MAMP in the presence of AMP. An Au disk electrode modified with AuNPs was applied to attach the aptamer sequence as the MAMP biocapture. By incubating the MAMP on the aptasensor surface and increasing the spatial restriction, the electron transfer of the redox probe (ferro/ferricyanide) was prevented from the electrode surface. The occurred signal-off corresponded to the MAMP concentration (Fig. 14) (Ebrahimi et al., 2012).

In another attempt, Rafiee and Fakhari took advantage of the synergistic effect of the MWCNTs, Nafion (Nf) and AuNPs to modify an SPE and prepare the SPE/MWCNTs-Nf/AuNPs as the MAMP sensor (Fig. 15). MAMP was captured by the framework created of MWCNTs and AuNPs on the modified SPE surface through the adsorption process. The electrochemical behavior of the MAMP on the sensor surface was monitored by both the square wave stripping voltammetry (SWSV) and EIS techniques. Based on the SWSV technique, two linear ranges $(20-100 \mathrm{nM}$ and 3-50 $\mu \mathrm{M})$ with a LOD of $6 \mathrm{nM}$ were achieved for the MAMP measurement. In contrast, the EIS technique presented a linear range of 1.15-26.9 nM with a lower LOD (0.3 nM) in MAMP detection (Rafiee et al., 2015).

The first impedimetric immunosensor in MAMP detection has been introduced by Yeh et al., in 2012. A conjugation of antibody-colloidal AuNPs as an electron bridge between two electrodes was applied to prepare the electro-microchip. Under a competitive immunoassay method, the MAMP led to the corresponding impedance signal change. This diagnostic method based on the electro-microchip has provided some advantages such as lower sample volume, fast analysis and the low concentration measurement with a LOD value of $7.13 \mathrm{nM}$ (Yeh et al., 2012).

\subsection{Amperometric sensors}

Amperometric sensors are usually applied to measure a current signal generated by an electrochemical reaction at a constant voltage (Hayat et al., 2014; Monroe 1990). This class of sensors has received remarkable attention due to high sensitivity and wide linear range in various sensing applications (Ran et al., 2010).

In 2002, Luangaram et al. developed the first amperometric immunosensor for MAMP detection. A combination of a carbon paste $(\mathrm{CP})$ and $\mathrm{Ag} / \mathrm{AgCl}$ screen printed on a heat-sealing film was used as an electrode. A composite of MAMP-N-BSA conjugate, antibody (as bioreceptor of MAMP) and alkaline phosphatase-goat anti-mouse immunoglobulin $G(\operatorname{IgG})$ were prepared under optimum ratio to detect MAMP. By conversion of p-aminophenyl phosphate to p-aminophenol, the MAMP was measured by the sensor in a urine sample under a competitive format. A linear range of 1.426-10.69 $\mu \mathrm{M}$ was reported (Luangaram et al., 2002).

Several years later, in 2014, Zhang and Liu presented another amperometric immunosensor in recognition of MAMP. An Au electrode (AuE) modified with the Prussian blue (PB), L-cystine (LC), nano-Au and (3-mercaptorpropyl) trimethoxysilane (MPS), which is denoted as the nano-Au/MPS/PB/LC/AuE, was fabricated. The PB was presented high activity toward the reduction of $\mathrm{H}_{2} \mathrm{O}_{2}$ and the corresponded signal enhanced. The measurement was done by capturing MAMP through the anti-MAMP under a dynamic range of 0.01-5 $\mu \mathrm{M}$ with a LOD of $7.5 \mathrm{nM}$ (Fig. 16) (Zhang and Liu 2014).

\subsection{Electrochemiluminetric sensors}

In the electrochemiluminescence or electrogenerated chemiluminescence $(\mathrm{ECL})$ process, the alteration of electrical energy into radiant energy accomplishes at an electrode surface by controlling the applied potential for the working electrode (Richter 2004). This process implicates the production of the species at the electrode surface to undergo a series of electron transfer reactions under specific conditions to create the excited states in the light emission (Richter 2004). Due to electrochemiluminetric sensors offer some advantages such as low background signal and suitable time and space control, they are significantly considered in the trace analysis of biological samples (Bard 2004; Delaney et al., 2011).

Up to now, various ECL sensors have been reported in the detection of MAMP such that most of them have utilized tris $\left(2,2^{\prime}-\right.$ bipyridine)ruthenium(II), $\mathrm{Ru}(\mathrm{bpy}) 3^{2+}$, as a chemiluminescence reagent. Changqing's group was introduced the first electrochemiluminetric sensor in MAMP detection in 2005. They applied tetramethoxysilane (TMOS) and dimethyldimethoxysilane (DiMe-DiMOS) as co-precursor in the attachment of poly(pstyrenesulfonate), PSS, to immobilize the $\mathrm{Ru}(\mathrm{bpy}) 3_{3}^{2+}$ on a GCE surface through ion-association. ECL characterizations of the modified electrodes with the organically modified silicates (ORMOSILs) were studied to measure MAMP by applying CV and chronocoulometry techniques. Based on the electron transfer mechanism between $\mathrm{Ru}(\mathrm{bpy}) 3_{3}^{2+}$ and MAMP during the ECL process (Scheme 5 ), the MAMP detection was done under a linear range of $0.5-1000 \mu \mathrm{M}$ with a LOD of $200 \mathrm{nM}$ (Yi et al., 2005).

Another application of the $\mathrm{Ru}(\mathrm{bpy}) 3_{3}^{2+}$ reagent in the ECL sensor has presented by Chen et al. in the MAMP assay. They utilized the synergistic effect of MWCNTs and ionic liquid to prepare a suitable nanocomposite in the immobilization of $\mathrm{Ru}(\mathrm{bpy}) 32+$. In this strategy, the ionic liquid as a conductive medium constructed an unblocked charge-transfer bridge to facilitate the intake of electrons from MWCNTs and Ru(bpy)32+. Additionally, the electrostatic interaction between the inner-sphere $\mathrm{Fe}(\mathrm{CN})_{6}^{3-} /{ }^{4-}$ couple as the probe and cation available in the ionic liquid structure improve the signal. By supporting some unique features such as high stability and excellent resistance to fouling, the sensor detected MAMP with a LOD of $8 \mathrm{nM}$ (Dai et al., 2009).

In another ECL sensor for the MAMP tracking, the synergic effect of the Ru(bpy)32+-doped SiNPs (Ru-SiNPs) and Nf was applied in the preparation of a nanocomposite to modify a GCE. The GCE modified with the Ru-SiNPs/Nf nanocomposite, which is denoted as a Ru-SiNPs/Nf/GCE, enlarged the active surface area of the electrode and embedded a fantastic platform for the MAMP oxidation. By oxidation of the MAMP, an intermediate was produced to react with $\mathrm{Ru}(\mathrm{bpy}) 3_{3}^{3+}$. The sensor analyzed MAMP with a LOD of $26 \mathrm{nM}$ (Cai 

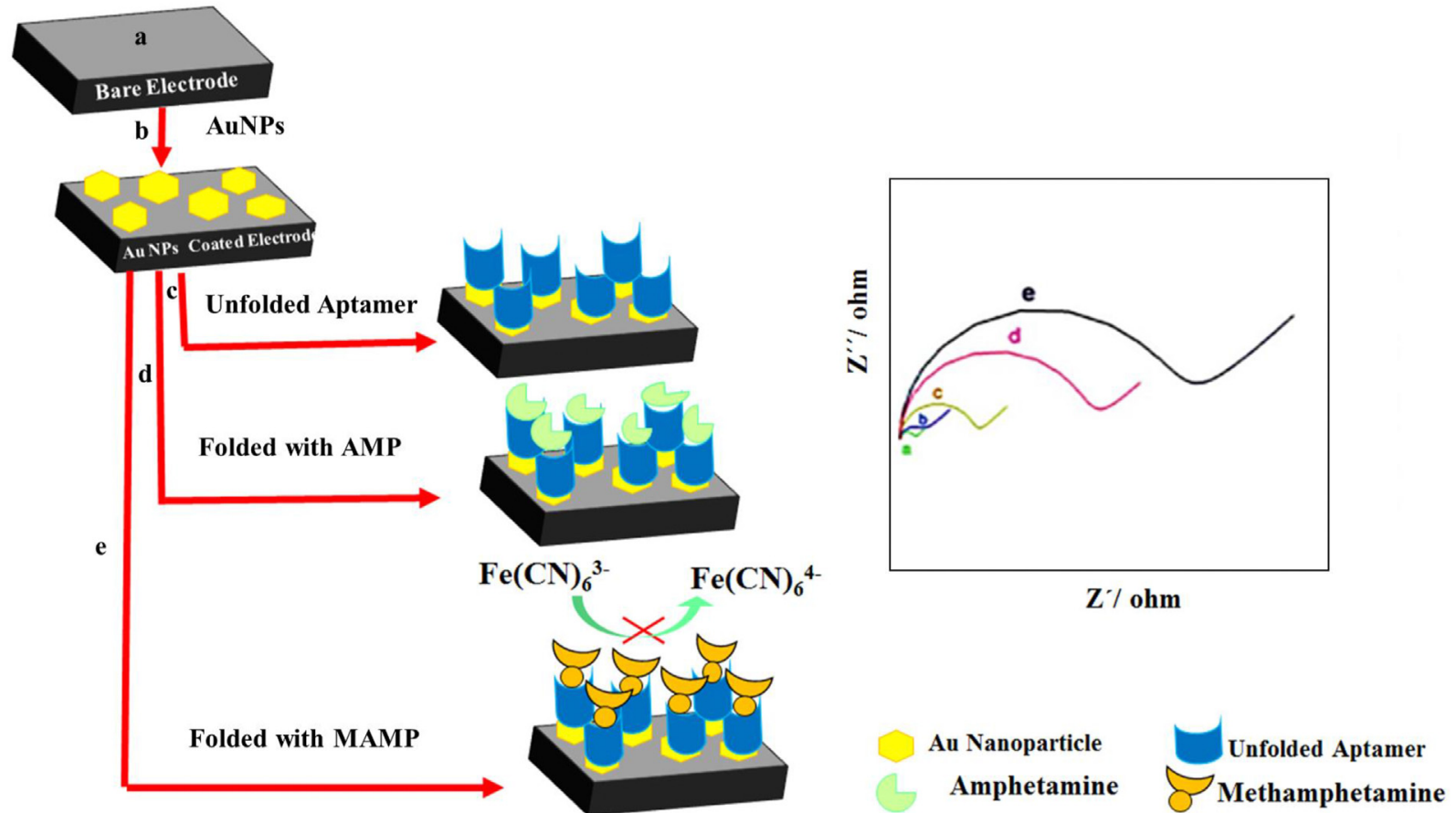

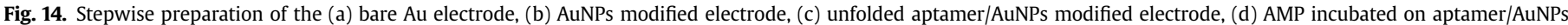

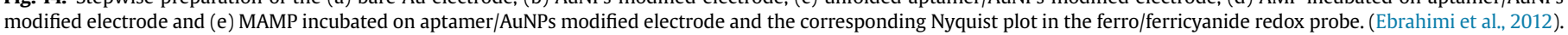

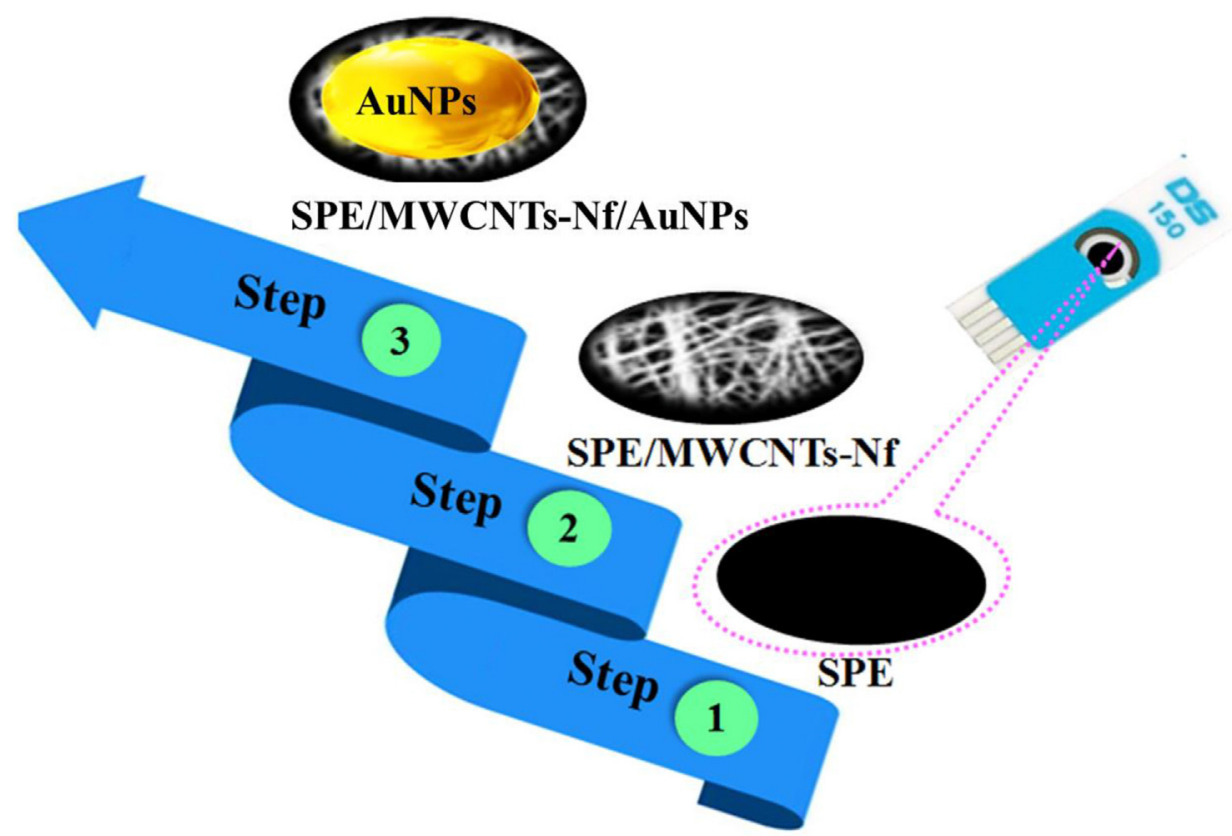

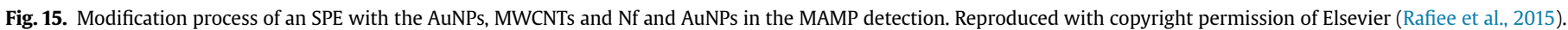

et al., 2010).

In a different study, an ECL immunosensor was fabricated to detect MAMP (Fig. 17). Lin et al. used indium tin oxide (ITO) coated glass covered with AuNPs via (3-aminopropyl)trimethoxysilane (APTMS) as a linker. The antibody was immobilized on AuNPs on the embedded ITO surface and luminol was utilized as an ECL agent to probe the interaction between the antibody and MAMP. The space hindrance was increased by incubating the MAMP on the surface, and the corresponding ECL intensity was decreased. The sensor detected MAMP under a linear range of 14.26-3.56 $\times 10^{3} \mathrm{nM}$ with a LOD of $2.14 \mathrm{nM}$ (Jiang et al., 2015).

Table 2 summarizes some sensors that have been reported electrochemical measuring of the MAMP.

\section{Comparing optical and electrochemical sensors in MAMP detection}

As discussed, the optical methods have been severally utilized to 

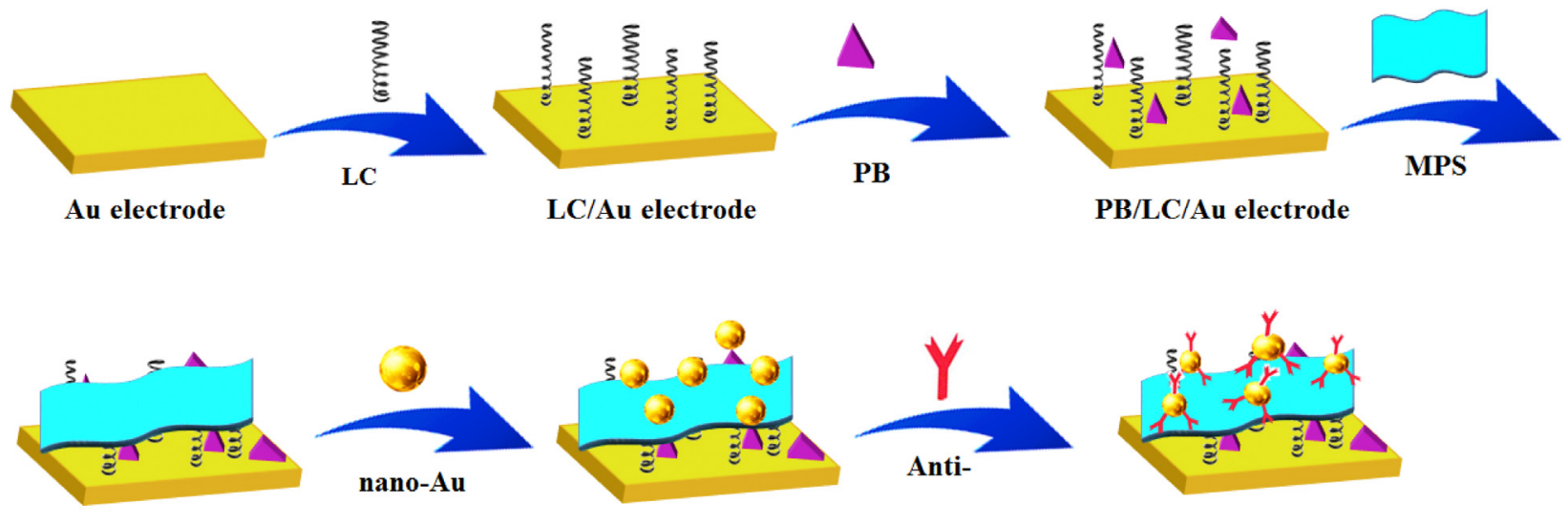

MPS/PB/LC/Au electrode

nano-Au/MPS/PB/LC/Au electrode

Anti-/nano-Au/MPS/PB/LC/Au electrode

Fig. 16. Schematically description of the Anti-/nano-Au/MPS/PB/LC electrode preparation procedure in the MAMP detection. Reproduced with copyright permission of Elsevier (Zhang and Liu 2014).

$$
\begin{aligned}
& \mathrm{Ru}(\mathrm{bpy})_{3}{ }^{2+} \longrightarrow \mathrm{Ru}(\mathrm{bpy})_{3}{ }^{3+}+\mathrm{e}^{-}
\end{aligned}
$$

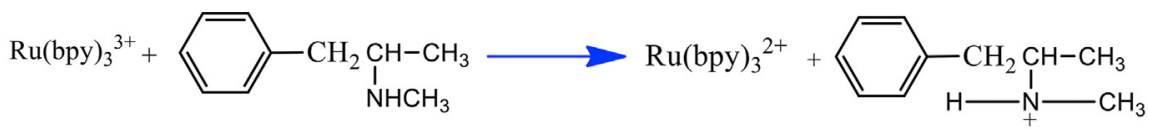

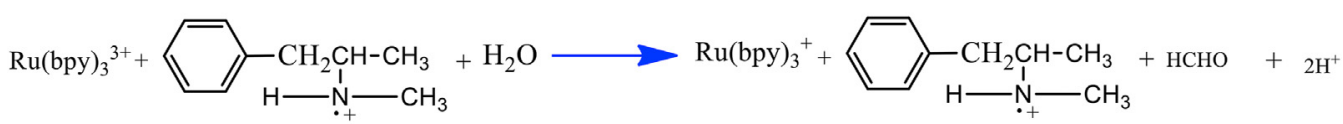

$$
\begin{aligned}
& \mathrm{Ru}(\mathrm{bpy})_{3}{ }^{+}+\mathrm{Ru}(\mathrm{bpy})_{3}{ }^{3+} \longrightarrow \mathrm{Ru}(\mathrm{bpy})_{3}{ }^{2+}+\mathrm{Ru}(\mathrm{bpy})_{3}{ }^{2+*} \\
& \mathrm{Ru}(\mathrm{bpy})_{3}{ }^{2+*} \longrightarrow \mathrm{Ru}(\mathrm{bpy})_{3}{ }^{2+}+\mathrm{hv}
\end{aligned}
$$

Scheme 5. The interaction of Ru(bpy) ${ }_{3}^{2+}$ with MAMP under the ECL process. Reproduced with copyright permission of Elsevier (Yi et al., 2005).

design MAMP sensors. While this sensing approach has presented great potential in highly sensitive MAMP monitoring, there have some pros and cons. All the advantages and disadvantages listed for optical sensors are corrected for the MAMP detection. The optical methods generally probe some differences created in scattering, absorbance, photoluminescence and chemiluminescence. They are chemically inert, immune to electromagnetic interference, suitable for remote sensing of the MAMP. Despite their benefits, some disadvantages include complexity, susceptibility to interference from environmental effects, or physical damage and require trained personnel to restrict their application. For example, the intrinsic fluorescence features of non-target molecules create some problems, such as background noises and false positives in fluorometric sensing of the MAMP. The overcoming this issue is needed some sample pretreatment steps to remove the interference of other molecules (Abu-Salah et al., 2015). During different years many attempts have been made to achieve optical MAMP sensing based on a simple and lightweight device by reliable operation without the need for trained personnel. Accordingly, various strategies have been applied based on aptamer, antibody and MIP integrated with an optical transducer to improve the analytical figures of merit. The nanomaterial-based optical sensors provide various sensing interfaces coupled with several spectroscopic techniques to present state-of-the-art sensing systems in MAMP tracking. Although these sensors have made advances in MAMP screening, their practical usage has been limited in clinical fields. Most of them are still in the initial levels of the principle illustrating (Abu-Salah et al., 2015; Pashchenko et al., 2018).

Since the electrochemical MAMP sensors rely on a redox reaction on a working electrode surface to produce an applicable qualitative or quantitative signal, they present some admirable features in the MAMP detection without any damage to the host system. This class of sensors with commercialization capability has specifically detected MAMP under wide linear dynamic range and low LOD. They are capable of miniaturizing and the experimental cost-reducing comparing to the optical-based sensor. Because the electrochemical MAMP sensors do not work based on the solution color change, unlike fluorometric or colorimetric methods, they are efficient for analyzing some non-clear samples such as blood. Additionally, some corresponding interferes of the light source have no limitation in this method (Abu-Salah et al., 2015). On the other hand, since the signal position change of anodic/cathodic peak is easily influenced, some intrinsic restrictions are made in this sensing strategy. Due to some electrode modifiers being degradated after several tests, their stability is decreased during the time. Various receptors such as the antibody, aptamer, or MIP eliminate some stability problems. The emergence of nanomaterials in the electrode modification process has opened new prospects in developing diverse strategies for electrochemical MAMP measurement. The various nanomaterials enhance the conductivity and surface area to facilitate the MAMP tracking under a relatively wide potential window in aqueous media with low cost 


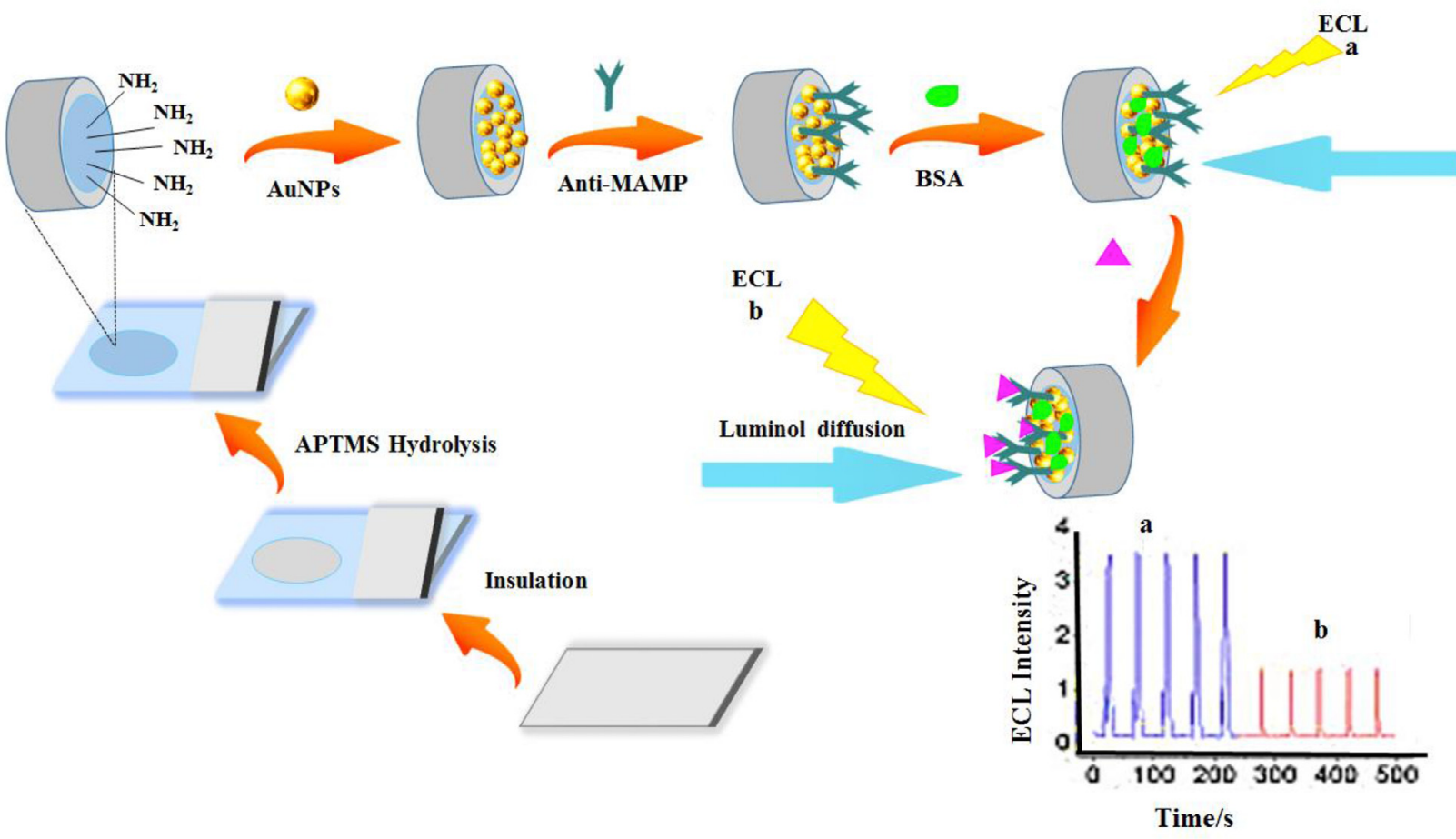

Fig. 17. Schematically fabrication the ECL sensor using the indium tin oxide (ITO) coated glass, APTMS and AuNPs to capture the Anti-MAMP underlying of the luminol as the ECL probe in (a) absence and (b) presence of the MAMP. Reproduced with copyright permission of Elsevier (Jiang et al., 2015).

Table 2

The electrochemical sensors for MAMP detection.

\begin{tabular}{|c|c|c|c|c|c|}
\hline Method & Modifier & Range $(\mu \mathrm{M})$ & LOD (nM) & Analyzed medium & Ref. \\
\hline Voltammetric (CV) & GCE & - & & Serum, Tablet & Garrido et al. (2010) \\
\hline Voltammetric (CV) & Aptamer/AuNPs/Chitosan/GCE & & 10 & & Kohzadi et al. (2016) \\
\hline Voltammetric (DPV) & BDDE & $0.07-80$ & 50 & Urine & Švorc et al. (2014) \\
\hline Voltammetric (DPV) & PGE & $74-54 \times 10^{3}$ & 50 & $\begin{array}{l}\text { Serum, Urine, Seized } \\
\text { drugs }\end{array}$ & Oghli et al. (2015) \\
\hline Voltammetric (DPV) & GCE/EDOT-BTDA-Pala/Antibody/MAMP & $71.31-713.1$ & 93.20 & Saliva, Serum, Urine & Demir et al. (2016) \\
\hline Voltammetric (SWV) & SPCE & & $2.85 \times 10^{-3}$ & Saliva & Bartlett et al. (2016) \\
\hline Voltammetric (FFT-SWV) & MIP/MWCNTs-CPE & $0.01-100$ & 0.83 & Serum, Urine & Akhoundian et al. (2019) \\
\hline Voltammetric (SWV) & $\begin{array}{l}\text { GCE/MWCNT/Au-NPs-SH- }\left(\mathrm{CH}_{2}\right)_{3}-\mathrm{Si} \\
-\mathrm{SiO}_{2} @ \mathrm{Fe}_{3} \mathrm{O}_{4}\end{array}$ & $0.05-50$ & 16 & Urine & (Haghighi et al.) \\
\hline Voltammetric (SWV) & $\mathrm{CeO}_{2} \mathrm{NP} / \mathrm{rGO} / \mathrm{GCE}$ & $25-166.6$ & $8.7 \times 10^{3}$ & Plasma & Anvari et al. (2021) \\
\hline Potentiometric & PVC-Membrane/NaTFPB & - & $10^{4}$ & Urine & Watanabe et al. (1993) \\
\hline Impedimetric & Aptamer/AuNPs/Au disk electrode & & & & Ebrahimi et al. (2012) \\
\hline Impedimetric & AuNPs/MWCNTs-Nf/SPE & $\begin{array}{l}1.15 \times 10^{-3} \\
-2.69 \times 10^{-2}\end{array}$ & 0.3 & - & Rafiee et al. (2015) \\
\hline Impedimetric & MAMP-antibody-colloidal Au conjugate & 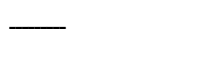 & $\begin{array}{l}356.55,0.71 \\
7.13 \times 10^{-3}\end{array}$ & - & Yeh et al. (2012) \\
\hline Impedimetric & ssDNA aptaMAMP & - & - & & Ebrahimi et al. (2013) \\
\hline Amperometric & SPCE/MAMP-BSA/AP-IgG & $1.42-10.69$ & 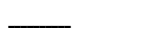 & Urine & Luangaram et al. (2002) \\
\hline Amperometric & GE/LC/PB/MPS/AuNP/Anti- MAMP & $0.01-5$ & 7.5 & Serum & Zhang and Liu (2014) \\
\hline Electrochemiluminescence & $\mathrm{Ru}(\mathrm{bpy}) 3_{3}^{2+} /$ Ormosils/GCE & $0.5-1000$ & 200 & Scout cases & Yi et al. (2005) \\
\hline Electrochemiluminescence & $\mathrm{Ru}(\mathrm{bpy}) 3_{3}^{2+} / \mathrm{MWCNTs}$-ionic liquid & $0.01-80$ & 8 & Urine & Dai et al. (2009) \\
\hline Electrochemiluminescence & GCE/RuSiNPs-Nafion Composite & $0.1-10$ & 26 & Drugs & Cai et al. (2010) \\
\hline Electrochemiluminescence & ITO/APTMS/AuNPs/Anti-MA & $14.26-3.56 \times 10^{3}$ & 2.14 & Serum & Jiang et al. (2015) \\
\hline Electrochemiluminescence & $\mathrm{Ru}(\mathrm{bpy})_{3}^{2+} /$ Nafion & $50 \times 10^{-6}-1000$ & $50 \times 10^{-3}$ & Street & $\begin{array}{l}\text { McGeehan and Dennany } \\
\text { (2016) }\end{array}$ \\
\hline Electrochemiluminescence & {$[\mathrm{Ru}(\mathrm{bpy}) 3]^{2+} / \mathrm{ATS}$ system } & - & - & Urine & Takahashi et al. (2018) \\
\hline
\end{tabular}

and low background current.

\section{Concluding remarks and future perspectives}

MAMP, as one of the most commonly misused stimulant drugs in the world, is a considerable threat to the health of the community. This growing worrying phenomenon is a contributing factor in severe social problems that should seriously be resolved. Since MAMP usually finds along with other street drugs, so an immediate and sensitive MAMP diagnosis in a mixture of stimulant drugs plays 
a critical role in supervision on drug use and acquiescence with treatment. For the first time, this review discussed how optical and electrochemical-based approaches are applied to develop efficient MAMP tracking sensors. The present state of the developed sensors shows relatively satisfactory progress have been made in this field. Of course, it is worth mentioning that both optical and electrochemical strategies have some pros and cons, but they have potentially facilitated the accurate MAMP assay. Nearly all MAMP sensing aspects have highlighted specific approaches due to the fantastic nanomaterial features. Some innovations have appeared in nanomaterial-based methods, although it seems clear that this area is ripe for many additional creative advances. However, the discussed strategies in this study have presented good benefits in MAMP recognition individually. As a significant drawback, it is still a challenge to construct a portable sensor array to analyze a mixture of several addictive substances. Whether using optical or electrochemical approaches in new methods for solving problems in simultaneous measurements of narcotic drugs, this field is exciting to continue to attract interest for a long time to come.

Nevertheless, this review exhibited various academic proof-ofconcept studies with significant advantages in the MAMP sense. However, there is still a knowledge gap between researchers and practitioners concerning constructing a valid sensor in the real world. The MAMP sensor commercialization for the routine analysis needs miniaturized devices based on the lab-on-a-chip technology to reduce the sensor cost for automatized surveillance. Considering the rapid expansion in the appearance of some materials as a receptor and integrating them with nanomaterials in the analytical approaches can be driven remarkable advance in the fabrication of sensor array to be marketed soon. The development of sensing devices based on an advanced nanomaterial, which can make hundreds and even thousands of measurements more rapid and cost-effective, will become available within the next decade. It is hoped that some of the provided insights can pave the way for new strategies in the construction of a cost-effective sensor array to detect other illicit drugs.

\section{Credit author statement}

Zeynab Khorablou: Written the paper, Dr. Faezeh Shahdost-fard and Prof. Habib Razmi: Editing of paper and checking of literature., Prof. Hassan Karimi-Maleh: Revision of paper.

\section{Declaration of competing interest}

The authors declare that they have no known competing financial interests or personal relationships that could have appeared to influence the work reported in this paper.

\section{Acknowledgments}

This study was supported by Azarbaijan Shahid Madani University and Farhangian University. Since this review was programmed and performed during the quarantine era of the Covid-19 pandemic, the authors dedicate this study to all the health personnel are working tirelessly to fight against Corona. Be well and safe.

\section{References}

Abu-Salah, K.M., Zourob, M.M., Mouffouk, F., Alrokayan, S.A., Alaamery, M.A., Ansari, A.A., 2015. DNA-based nanobiosensors as an emerging platform for detection of disease. Sensors 15 (6), 14539-14568.

Adams, K., Adams, T., Aldworth, J., Asman, K., Barnett, S., Bishop, E., 2016. Results from the 2016 National Survey on Drug Use and Health: Detailed Tables. Center for Behavioral Health Statistics and Quality (CBHSQ), Substance Abuse and
Mental Health Services Administration (SAMHSA). US Department of Health and Human Services (HHS), RTI International.

Akhoundian, M., Alizadeh, T., Ganjali, M.R., Norouzi, P., 2019. Ultra-trace detection of methamphetamine in biological samples using FFT-square wave voltammetry and nano-sized imprinted polymer/MWCNTs-modified electrode. Talanta 200, $115-123$.

Alizadeh, N., Mohammadi, A., Tabrizchi, M., 2008. Rapid screening of methamphetamines in human serum by headspace solid-phase microextraction using a dodecylsulfate-doped polypyrrole film coupled to ion mobility spectrometry. J. Chromatogr. 1183 (1-2), 21-28.

Anglin, M.D., Burke, C., Perrochet, B., Stamper, E., Dawud-Noursi, S., 2000. History of the methamphetamine problem. J. Psychoact. Drugs 32 (2), 137-141.

Anvari, L., Ghoreishi, S.M., Faridbod, F., Ganjali, M.R., 2021. Electrochemical determination of methamphetamine in human plasma on a nanoceria nanoparticle decorated reduced graphene oxide (rGO) glassy carbon electrode (GCE). Anal. Lett. 1-14.

Argente-García, A., Jornet-Martínez, N., Herráez-Hernández, R., Campíns-Falcó, P., 2016. A solid colorimetric sensor for the analysis of amphetamine-like street samples. Anal. Chim. Acta 943, 123-130.

Baghbamidi, S.E., Beitollahi, H., Tajik, S., Hosseinzadeh, R., 2016. Voltammetric sensor based on 1-benzyl-4-ferrocenyl-1H-[1, 2, 3]-triazole/carbon nanotube modified glassy carbon electrode; detection of hydrochlorothiazide in the presence of propranolol. International Journal of Electrochemical Science 11 (12), 10874-10883.

Bard, A.J., 2004. Electrogenerated Chemiluminescence. CRC Press.

Bartlett, C.-A., Taylor, S., Fernandez, C., Wanklyn, C., Burton, D., Enston, E., Raniczkowska, A., Black, M., Murphy, L., 2016. Disposable screen printed sensor for the electrochemical detection of methamphetamine in undiluted saliva. Chem. Cent. J. 10 (1), 3.

Bockris, J.O.M., 1981. xviii- 718 pp.£ 14.70 Electrochemical Methods-Fundamentals and Applications, Allen J. Bard, Larry R. Faulkner, 1980. Wiley, New York. Elsevier.

Cai, Z., Lin, Z., Chen, X., Jia, T., Yu, P., Chen, X., 2010. Electrochemiluminescence detection of methamphetamine based on a Ru (bpy) 32+-doped silica nanoparticles/Nafion composite film modified electrode. Luminescence 25 (5), 367-372.

Cao, F., Xu, J., Yan, S., Yuan, X., Yang, F., Hou, L., Zhao, L., Zeng, L., Liu, W., Zhu, L., 2018. A surface plasmon resonance-based inhibition immunoassay for forensic determination of methamphetamine in human serum. Forensic Chemistry 8 , $21-27$.

Chang T.-C., Chiang C.-Y, Lin, M.-H., Chen, I.-K, Chau, L.-K. Hsu, D.-S, Shieh, S.-S., Kuo, C.-J., Wang, S.-C., Chen, Y.-f., 2020. Fiber optic particle plasmon resonance immunosensor for rapid and sensitive detection of methamphetamine based on competitive inhibition. Microchem. J. 105026.

Cheng, Q., Tang, J., Ma, J., Zhang, H., Shinya, N., Qin, L.-C., 2011. Polyaniline-coated electro-etched carbon fiber cloth electrodes for supercapacitors. J. Phys. Chem. C 115 (47), 23584-23590.

Chinaka, S., Tanaka, S., Takayama, N., Komai, K., Ohshima, T., Ueda, K. 2000. Simultaneous chiral analysis of methamphetamine and related compounds by capillary electrophoresis. J. Chromatogr. B Biomed. Sci. Appl. 749 (1), 111-118.

Chou, C.-C., Lee, M.-R., 2005. Solid phase microextraction with liquid chromatography-electrospray ionization-tandem mass spectrometry for analysis of amphetamine and methamphetamine in serum. Anal. Chim. Acta $538(1-2), 49-56$.

Dai, H., Wang, Y., Wu, X., Zhang, L., Chen, G., 2009. An electrochemiluminescent sensor for methamphetamine hydrochloride based on multiwall carbon nanotube/ionic liquid composite electrode. Biosens. Bioelectron. 24 (5), 1230-1234.

Dandin, M., Abshire, P., Smela, E., 2007. Optical filtering technologies for integrated fluorescence sensors. Lab Chip 7 (8), 955-977.

Delaney, J.L., Hogan, C.F., Tian, J., Shen, W., 2011. Electrogenerated chemiluminescence detection in paper-based microfluidic sensors. Anal. Chem. 83 (4), 1300-1306.

Demir, B., Yilmaz, T., Guler, E., Gumus, Z.P., Akbulut, H., Aldemir, E., Coskunol, H., Colak, D.G., Cianga, I., Yamada, S., 2016. Polypeptide with electroactive endgroups as sensing platform for the abused drug 'methamphetamine'by bioelectrochemical method. Talanta 161, 789-796.

Dennis, A.M., Rhee, W.J., Sotto, D., Dublin, S.N., Bao, G., 2012. Quantum dot-fluorescent protein FRET probes for sensing intracellular pH. ACS Nano 6 (4), 2917-2924.

Djozan, D., Farajzadeh, M.A., Sorouraddin, S.M., Baheri, T., 2012. Molecularly imprinted-solid phase extraction combined with simultaneous derivatization and dispersive liquid-liquid microextraction for selective extraction and preconcentration of methamphetamine and ecstasy from urine samples followed by gas chromatography. J. Chromatogr. 1248, 24-31.

Donaldson, M., Goodchild, J.H., 2006. Oral health of the methamphetamine abuser Am. J. Health Syst. Pharm. 63 (21), 2078-2082.

Dong, R., Weng, S., Yang, L., Liu, J., 2015. Detection and direct readout of drugs in human urine using dynamic surface-enhanced Raman spectroscopy and support vector machines. Anal. Chem. 87 (5), 2937-2944.

Dubertret, B., Skourides, P., Norris, D.J., Noireaux, V., Brivanlou, A.H., Libchaber, A., 2002. In vivo imaging of quantum dots encapsulated in phospholipid micelles. Science 298 (5599), 1759-1762.

Ebrahimi, M., Hamzeiy, H., Barar, J., Barzegari, A., Omidi, Y., 2013. Systematic evolution of ligands by exponential enrichment selection of specific aptamer for 
sensing of methamphetamine. Sens. Lett. 11 (3), 566-570.

Ebrahimi, M., Johari-Ahar, M., Hamzeiy, H., Barar, J., Mashinchian, O., Omidi, Y., 2012. Electrochemical impedance spectroscopic sensing of methamphetamine by a specific aptamer. Bioimpacts: BI 2 (2), 91.

Fang, W., Zhang, B., Han, F.-Y., Qin, Z.-N., Feng, Y.-Q., Hu, J.-M., Shen, A.-G., 2020. Onsite and quantitative detection of trace methamphetamine in urine/serum samples with a surface-enhanced Raman scattering-active microcavity and rapid pretreatment device. Anal. Chem. 92 (19), 13539-13549.

Foroughi, M.M., Beitollahi, H., Tajik, S., Akbari, A., Hosseinzadeh, R., 2014. Electrochemical determination of $\mathrm{N}$-acetylcysteine and folic acid in pharmaceutical and biological samples using a modified carbon nanotube paste electrode. Int. J. Electrochem 9, 8407.

Freire, R.S., Pessoa, C.A., Mello, L.D., Kubota, L.T., 2003. Direct electron transfer: an approach for electrochemical biosensors with higher selectivity and sensitivity. J. Braz. Chem. Soc. 14 (2), 230-243.

Fu, Y., Shi, L., Zhu, D., He, C., Wen, D., He, Q., Cao, H., Cheng, J., 2013. Fluorene-thiophene-based thin-film fluorescent chemosensor for methamphetamine vapor by thiophene-amine interaction. Sensor. Actuator. B Chem. 180, $2-7$.

Ganjali, M.R. Salimi, H., Tajik, S., Beitollahi, H., Rezapour, M., Larijani, B., 2017. Application of Fe304@SiO2/MWCNT film on glassy carbon electrode for the sensitive electroanalysis of levodopa. International Journal of Electrochemical Science 12 (6), 5243-5253.

Gao, X., Cui, Y., Levenson, R.M., Chung, L.W., Nie, S., 2004. In vivo cancer targeting and imaging with semiconductor quantum dots. Nat. Biotechnol. 22 (8), 969-976.

Garrido, E., Garrido, J., Milhazes, N., Borges, F., Oliveira-Brett, A., 2010. Electrochemical oxidation of amphetamine-like drugs and application to electroanalysis of ecstasy in human serum. Bioelectrochemistry 79 (1), 77-83.

Guoning, C., Pengqi, G., Yan, W., Lu, W., Hua, S., Yunzhe, L., Wanghui, J., Chun, C., Qiang, F., 2019. Preparation of molecularly imprinted polymers and application in a biomimetic biotin-avidin-ELISA for the detection of bovine serum albumin. Talanta 198, 55-62.

Haghighi, M., Shahlaei, M., Irandoust, M., Hassanpour, A., New and Sensitive Sensor for Voltammetry Determination of Methamphetamine in Biological Samples.

Hassanzadeh, J., Khataee, A., Lotfi, R., 2017. Sensitive fluorescence and chemiluminescence procedures for methamphetamine detection based on CdS quantum dots. Microchem. J. 132, 371-377.

Haupt, K., 2001. Molecularly imprinted polymers in analytical chemistry. Analyst 126 (6), 747-756.

Haupt, K., Mosbach, K., 2000. Molecularly imprinted polymers and their use in biomimetic sensors. Chem. Rev. 100 (7), 2495-2504.

Hayat, A., Catanante, G., Marty, J.L., 2014. Current trends in nanomaterial-based amperometric biosensors. Sensors 14 (12), 23439-23461.

Homola, J., 2008. Surface plasmon resonance sensors for detection of chemical and biological species. Chem. Rev. 108 (2), 462-493.

Hong, Y., Zhou, X., Xu, B., Huang, Y., He, W., Wang, S., Wang, C., Zhou, G., Chen, Y., Gong, T., 2020. Optoplasmonic hybrid materials for trace detection of methamphetamine in biological fluids through SERS. ACS Appl. Mater. Interfaces 12 (21), 24192-24200.

Jiang, L., Yang, Y., Tu, Y., 2015. A new strategy to develop the disposable label-free immunosensor with electrochemiluminescent probing. J. Electroanal. Chem. $747,136-142$.

Kahl, J.M.M., da Cunha, K.F., Rodrigues, L.C., de Oliveira Chinaglia, K., Oliveira, K.D., Costa, J.L., 2021. Quantification of amphetamine and derivatives in oral fluid by dispersive liquid-liquid microextraction and liquid chromatography-tandem mass spectrometry. J. Pharmaceut. Biomed. Anal., 113928

Karila, L., Weinstein, A., Aubin, H.J., Benyamina, A., Reynaud, M., Batki, S.L., 2010. Pharmacological approaches to methamphetamine dependence: a focused review. Br. J. Clin. Pharmacol. 69 (6), 578-592.

Karim-Nezhad, G., Khorablou, Z., Dorraji, P.S., 2016. Modification of glassy carbon electrode with a bilayer of multiwalled carbon nanotube/poly (l-arginine) in the presence of surfactant: application to discrimination and simultaneous electrochemical determination of dihydroxybenzene isomers. J. Electrochem. Soc. 163 (7), B358-B365.

Karim-Nezhad, G., Khorablou, Z., Dorraji, P.S., 2018. A promising electrochemical sensing platform based on copper nanoparticles-decorated polymer in carbon nanotube electrode for monitoring methimazole. J. Iran. Chem. Soc. 15 (4), 905-913.

Karimi-Maleh, H., Alizadeh, M., Orooji, Y., Karimi, F., Baghayeri, M., Rouhi, J., Tajik, S., Beitollahi, H., Agarwal, S., Gupta, V.K., 2021a. Guanine-based DNA biosensor amplified with Pt/SWCNTs nanocomposite as analytical tool for nanomolar determination of daunorubicin as an anticancer drug: a docking/experimental investigation. Ind. Eng. Chem. Res. 60 (2), 816-823.

Karimi-Maleh, H., Yola, M.L., Atar, N., Orooji, Y., Karimi, F., Kumar, P.S., Rouhi, J., Baghayeri, M., 2021b. A novel detection method for organophosphorus insecticide fenamiphos: molecularly imprinted electrochemical sensor based on core-shell Co304@ MOF-74 nanocomposite. J. Colloid Interface Sci. 592, $174-185$.

Karimi-Maleh, H., Karimi, F., Alizadeh, M., Sanati, A.L., 2020. Electrochemical sensors, a bright future in the fabrication of portable kits in analytical systems. Chem. Rec. 20 (7), 682-692.

Kim, Y.S., Kim, J.H., Kim, I.A., Lee, S.J., Jurng, J., Gu, M.B., 2010. A novel colorimetric aptasensor using gold nanoparticle for a highly sensitive and specific detection of oxytetracycline. Biosens. Bioelectron. 26 (4), 1644-1649.
Kohzadi, R., Molaeirad, A., Alijanianzadeh, M., Kamali, N., Mohtashamifar, M., 2016. Designing a label free aptasensor for detection of methamphetamine. Biomacromolecular Journal 2 (1), 28-33.

Kumihashi, M., Ameno, K., Shibayama, T., Suga, K., Miyauchi, H., Jamal, M., Wang, W. Uekita, I., Ijiri, I., 2007. Simultaneous determination of methamphetamine and its metabolite, amphetamine, in urine using a high performance liquid chromatography column-switching method. J. Chromatogr. B 845 (1), 180-183.

Li, B., Wang, T., Su, Q., Wu, X., Dong, P., 2019. Fabrication of Au nanorods by the Oblique angle deposition process for trace detection of methamphetamine with surface-enhanced Raman scattering. Sensors 19 (17), 3742.

Lobnik, A., Turel, M., Urek, Š., 2012. Optical Chemical Sensors: Design and Applications. InTech Rijeka, Croatia.

Lovrić, M., 2010. Square-wave Voltammetry. Electroanalytical Methods. Springer, pp. $121-145$.

Luangaram, K., Boonsua, D., Soontornchai, S., Promptmas, C., 2002. Development of an amperometric immunosensor for the determination of methamphetamine in urine. Biocatal. Biotransform. 20 (6), 397-403.

Mandani, S., Rezaei, B., Ensafi, A.A., 2020. Sensitive imprinted optical sensor based on mesoporous structure and green nanoparticles for the detection of methamphetamine in plasma and urine. Spectrochim. Acta Mol. Biomol. Spectrosc., 118077

Mansur, H.S., Mansur, A.A., González, J., 2011. Synthesis and characterization of CdS quantum dots with carboxylic-functionalized poly (vinyl alcohol) for bioconjugation. Polymer 52 (4), 1045-1054.

Mao, J., Kang, Y., Yu, D., Zhou, J., 2021. Surface-enhanced Raman spectroscopy integrated with aligner mediated cleavage strategy for ultrasensitive and selective detection of methamphetamine. Anal. Chim. Acta 1146, 124-130.

Mao, K., Yang, Z., Du, P., Xu, Z., Wang, Z., Li, X., 2016. G-quadruplex-hemin DNAzyme molecular beacon probe for the detection of methamphetamine. RSC Adv. 6 (67), 62754-62759.

Mao, K., Yang, Z., Li, J., Zhou, X., Li, X., Hu, J., 2017. A novel colorimetric biosensor based on non-aggregated Au@ Ag core-shell nanoparticles for methamphetamine and cocaine detection. Talanta 175, 338-346.

Mao, K., Zhou, Z., Han, S., Zhou, X., Hu, J., Li, X., Yang, Z., 2018. A novel biosensor based on Au@Ag core-shell nanoparticles for sensitive detection of methylamphetamine with surface enhanced Raman scattering. Talanta 190, 263-268.

Masteri-Farahani, M., Askari, F., 2019. Design and photophysical insights on graphene quantum dots for use as nanosensor in differentiating methamphetamine and morphine in solution. Spectrochim. Acta Mol. Biomol. Spectrosc. 206, 448-453.

Masteri-Farahani, M., Mashhadi-Ramezani, S., Mosleh, N., 2020. Molecularly imprinted polymer containing fluorescent graphene quantum dots as a new fluorescent nanosensor for detection of methamphetamine. Spectrochim. Acta Mol. Biomol. Spectrosc. 229, 118021.

Masteri-Farahani, M., Mosleh, N., 2019. Modified CdS quantum dots as selective turn-on fluorescent nanosensor for detection and determination of methamphetamine. J. Mater. Sci. Mater. Electron. 30 (24), 21170-21176.

McGeehan, J., Dennany, L., 2016. Electrochemiluminescent detection of methamphetamine and amphetamine. Forensic Sci. Int. 264, 1-6.

Meijerink, A., 2008. Semiconductor Nanocrystal Quantum Dots: Synthesis, Assembly, Spectroscopy and Applications. Springer, Nova York.

Miura, N., Higobashi, H., Sakai, G., Takeyasu, A., Uda, T., Yamazoe, N., 1993. Piezoelectric crystal immunosensor for sensitive detection of methamphetamine (stimulant drug) in human urine. Sensor. Actuator. B Chem. 13 (1-3), 188-191.

Miyazaki, C.M., Shimizu, F.M., Ferreira, M., 2017. Surface Plasmon Resonance (SPR) for Sensors and Biosensors. Nanocharacterization Techniques. Elsevier, pp. $183-200$.

Mohammad-Rezaei, R., Razmi, H., 2012. Reduced graphene Oxide| carbon ceramic electrode modified with CdS-hemoglobin as a sensitive hydrogen peroxide biosensor. Electroanalysis 24 (11), 2094-2101.

Monroe, D., 1990. Amperometric immunoassay. Crit. Rev. Clin. Lab Sci. 28 (1), 1-18.

Mooney, L., Glasner-Edwards, S., Rawson, R.A., Ling, W., 2009. Medical Effects of Methamphetamine Use. Methamphetamine Addiction: from Basic Science to Treatment, pp. 117-142.

Moshirian-Farahi, S.S., Zamani, H.A., Abedi, M., 2020. Nano-molar level determination of isoprenaline in pharmaceutical and clinical samples; A nanostructure electroanalytical strategy. Eurasian Chemical Communications 2 (6), 702-711.

Nuntawong, N., Eiamchai, P., Somrang, W., Denchitcharoen, S., Limwichean, S., Horprathum, M., Patthanasettakul, V., Chaiya, S., Leelapojanaporn, A. Saiseng, S., 2017. Detection of methamphetamine/amphetamine in human urine based on surface-enhanced Raman spectroscopy and acidulation treatments. Sensor. Actuator. B Chem. 239, 139-146.

Nutiu, R., Li, Y., 2005. In vitro selection of structure-switching signaling aptamers. Angew. Chem. Int. Ed. 44 (7), 1061-1065.

Oghli, A.H., Alipour, E., Asadzadeh, M., 2015. Development of a novel voltammetric sensor for the determination of methamphetamine in biological samples on the pretreated pencil graphite electrode. RSC Adv. 5 (13), 9674-9682.

Osteryoung, J.G., Osteryoung, R.A., 1985. Square wave voltammetry. Anal. Chem. 57 (1), 101-110.

Pan, J., Cha, T.-G., Chen, H., Choi, J., 2013. Carbon Nanotube-Based Optical Platforms for Biomolecular Detection. Carbon Nanotubes and Graphene for Photonic Applications. Elsevier, pp. 270-303e.

Parsons, N.L., 2014. Meth Mania: A History of Methamphetamine. Lynne Rienner Publishers, Incorporated.

Pashazadeh, P., Mokhtarzadeh, A., Hasanzadeh, M., Hejazi, M., Hashemi, M., de la 
Guardia, M., 2017. Nano-materials for use in sensing of salmonella infections: recent advances. Biosens. Bioelectron. 87, 1050-1064.

Pashchenko, O., Shelby, T., Banerjee, T., Santra, S., 2018. A comparison of optical, electrochemical, magnetic, and colorimetric point-of-care biosensors for infectious disease diagnosis. ACS Infect. Dis. 4 (8), 1162-1178.

Pérez-Fernández, B., Costa-García, A., Muñiz, A.d.l.E., 2020. Electrochemical (bio) sensors for pesticides detection using screen-printed electrodes. Biosensors 10 (4), 32.

Petryayeva, E., Krull, U.J., 2011. Localized surface plasmon resonance: nanostructures, bioassays and biosensing - a review. Anal. Chim. Acta 706 (1), 8-24.

Pickup, J.C., Hussain, F., Evans, N.D., Rolinski, O.J., Birch, D.J., 2005. Fluorescencebased glucose sensors. Biosens. Bioelectron. 20 (12), 2555-2565.

Piliarik, M., Vaisocherová, H., Homola, J., 2007. Towards parallelized surface plasmon resonance sensor platform for sensitive detection of oligonucleotides. Sensor. Actuator. B Chem. 121 (1), 187-193.

Prakash, M.D., Tangalakis, K., Antonipillai, J., Stojanovska, L., Nurgali, K., Apostolopoulos, V., 2017. Methamphetamine: effects on the brain, gut and immune system. Pharmacol. Res. 120, 60-67.

Rafiee, B., Fakhari, A.R., Ghaffarzadeh, M., 2015. Impedimetric and stripping voltammetric determination of methamphetamine at gold nanoparticlesmultiwalled carbon nanotubes modified screen printed electrode. Sensor. Actuator. B Chem. 218, 271-279.

Ragavan, K., Rastogi, N.K., Thakur, M., 2013. Sensors and biosensors for analysis of bisphenol-A. Trac. Trends Anal. Chem. 52, 248-260.

Ran, X.-Q., Yuan, R., Chai, Y.-Q., Hong, C.-L., Qian, X.-Q., 2010. A sensitive amperometric immunosensor for alpha-fetoprotein based on carbon nanotube/DNA/ Thi/nano-Au modified glassy carbon electrode. Colloids Surf. B Biointerfaces 79 (2), 421-426.

Razmi, H., Ezzati, L., Khorablou, Z., 2019. Direct electrochemical synthesis of graphene oxide/cobalt oxide nanocomposite on pencil graphite electrode for highly sensitive and selective detection of insulin in pharmaceutical samples. J. Electrochem. Soc. 166 (12), B961-B968.

Razmi, H., Mohammad-Rezaei, R., 2013. Graphene quantum dots as a new substrate for immobilization and direct electrochemistry of glucose oxidase: application to sensitive glucose determination. Biosens. Bioelectron. 41, 498-504.

Razmi, H., Sarhang-Zadeh, K., Mohammad-Rezaei, R., 2013. Electrochemical behavior and voltammetric determination of diclofenac at a multi-walled carbon nanotube-ionic liquid composite modified carbon ceramic electrode. Anal. Lett. 46 (12), 1885-1896.

Rezaei, B., Irannejad, N., 2019. Electrochemical Detection Techniques in Biosensor Applications. Electrochemical Biosensors. Elsevier, pp. 11-43.

Richter, M.M., 2004. Electrochemiluminescence (ecl). Chem. Rev. 104 (6), 3003-3036.

Rosy, S., Yadav, B.A., 2014. M. Oyama, RN Goyal, Graphene modified Palladium sensor for electrochemical analysis ofnorepinephrine in pharmaceuticals and biological fluids. Electrochim. Acta 125, 622-629.

Rouhani, S., Haghgoo, S., 2015. A novel fluorescence nanosensor based on 1, 8naphthalimide-thiophene doped silica nanoparticles, and its application to the determination of methamphetamine. Sensor. Actuator. B Chem. 209, 957-965.

Roushani, M., Dizajdizi, B.Z., Salimi, A., Azadbakht, A., 2019. Preparation of modified glassy carbon electrode by the use of titanium oxide, copper and palladium nanoparticles and its application for the electrocatalytic and photelectrocatalytic reduction of hydrogen peroxide. J. Mater. Sci. Mater. Electron. 30 (5), $5212-5221$.

Roushani, M., Ghanbarzadeh, M., Shahdost-Fard, F., Sahraei, R., Soheyli, E., 2020. AgNPs/QDs@GQDs nanocomposites developed as an ultrasensitive impedimetric aptasensor for ractopamine detection. Mater. Sci. Eng. C 108, 110507.

Roushani, M., Shahdost-fard, F., 2015. A highly selective and sensitive cocaine aptasensor based on covalent attachment of the aptamer-functionalized AuNPs onto nanocomposite as the support platform. Anal. Chim. Acta 853, 214-221.

Roushani, M., Shahdost-fard, F., 2017. Ultra-sensitive detection of ibuprofen (IBP) by electrochemical aptasensor using the dendrimer-quantum dot (Den-QD) bioconjugate as an immobilization platform with special features. Mater. Sci. Eng. C 75, 1091-1096.

Roushani, M., Shahdost-fard, F., 2018. Impedimetric detection of cocaine by using an aptamer attached to a screen printed electrode modified with a dendrimer/ silver nanoparticle nanocomposite. Microchimica Acta 185 (4), 214.

Roushani, M., Shahdost-Fard, F., 2019. Applicability of AuNPs@ N-GQDs nanocomposite in the modeling of the amplified electrochemical Ibuprofen aptasensing assay by monitoring of riboflavin. Bioelectrochemistry 126, 38-47.

Ruedas-Rama, M.J., Walters, J.D., Orte, A., Hall, E.A., 2012. Fluorescent nanoparticles for intracellular sensing: a review. Anal. Chim. Acta 751, 1-23.

Rusyniak, D.E., 2013. Neurologic manifestations of chronic methamphetamine abuse. Psychiatr. Clin. 36 (2), 261-275.

Saberi, Z., Rezaei, B., Faroukhpour, H., Ensafi, A.A., 2018. A fluorometric aptasensor for methamphetamine based on fluorescence resonance energy transfer using cobalt oxyhydroxide nanosheets and carbon dots. Microchimica Acta 185 (6), 303.

Sakai, G., Nakata, S., Uda, T., Miura, N., Yamazoe, N., 1999. Highly selective and sensitive SPR immunosensor for detection of methamphetamine. Electrochim. Acta 44 (21-22), 3849-3854.

Salemmilani, R., Piorek, B.D., Mirsafavi, R.Y., Fountain III, A.W., Moskovits, M., Meinhart, C.D., 2018. Dielectrophoretic nanoparticle aggregation for ondemand surface enhanced Raman spectroscopy analysis. Anal. Chem. 90 (13),
$7930-7936$.

Segawa, H., Okada, Y., Yamamuro, T., Kuwayama, K., Tsujikawa, K., Kanamori, T., Iwata, Y.T., 2021. Stereoselective analysis of ephedrine and its stereoisomers as impurities and/or by-products in seized methamphetamine by supercritical fluid chromatography/tandem mass spectrometry. Forensic Sci. Int. 318, 110591.

Shahdost-fard, F., Fahimi-Kashani, N., Hormozi-nezhad, M., A ratiometric fluorescence nanoprobe using CdTe QDs for fast detection of carbaryl insecticide in apple. Talanta 221, 121467.

Shahdost-fard, F., Roushani, M., 2016. Conformation switching of an aptamer based on cocaine enhancement on a surface of modified GCE. Talanta 154, 7-14.

Shahdost-fard, F., Roushani, M., 2017. The use of a signal amplification strategy for the fabrication of a TNT impedimetric nanoaptasensor based on electrodeposited NiONPs immobilized onto a GCE surface. Sensor. Actuator. B Chem. 246, 848-853.

Shahdost-fard, F., Roushani, M., 2019. Designing of an Ultrasensitive BCM-7 Aptasensor Based on an SPCE Modified with AuNR for Promising Distinguishing of Autism Disorder. Talanta, 120506.

Shahdost-fard, F., Roushani, M., 2020. Architecting of a biodevice based on a screenprinted carbon electrode modified with the NiONP nanolayer and aptamer in BCM-7 detection. Colloids and Surfaces B: Biointerfaces, 110932.

Shakeri, J., Farnia, V., Davarinejad, O., Salemi, S., Golshani, S., Rahami, B., Alikhani, M., Hookari, S., 2019. Distress Tolerance in Methamphetamine and Opium Abusers with Non-drug Abuser (A Comparative Analysis). Clinical Epidemiology and Global Health.

Shaner, J.W., Kimmes, N., Saini, T., Edwards, P., 2006. Meth mouth": rampant caries in methamphetamine abusers. AIDS Patient Care STDS 20 (3), 146-150.

Shen, J., Zhu, Y., Chen, C., Yang, X., Li, C., 2011. Facile preparation and upconversion luminescence of graphene quantum dots. Chem. Commun. 47 (9), 2580-2582.

Shen, J., Zhu, Y., Yang, X., Li, C., 2012. Graphene quantum dots: emergent nanolights for bioimaging, sensors, catalysis and photovoltaic devices. Chem. Commun. 48 (31), 3686-3699.

Shi, Q., Shi, Y., Pan, Y., Yue, Z., Zhang, H., Yi, C., 2015. Colorimetric and bare eye determination of urinary methylamphetamine based on the use of aptamers and the salt-induced aggregation of unmodified gold nanoparticles. Microchimica Acta 182 (3-4), 505-511.

Si, Y., 2015. Fluorescent Nanomaterials for Bioimaging and Biosensing: Application on E. coli Bacteria.

Skoog, D.A., Holler, F.J., Crouch, S.R., 2017. Principles of Instrumental Analysis. Cengage learning.

Sulk, R.A., Corcoran, R.C., Carron, K.T., 1999. Surface-enhanced Raman scattering detection of amphetamine and methamphetamine by modification with 2mercaptonicotinic acid. Appl. Spectrosc. 53 (8), 954-959.

Švorc, Ľ., Vojs, M., Michniak, P., Marton, M., Rievaj, M., Bustin, D., 2014. Electrochemical behavior of methamphetamine and its voltammetric determination in biological samples using self-assembled boron-doped diamond electrode. J. Electroanal. Chem. 717, 34-40.

Taghdisi, S.M., Danesh, N.M., Ramezani, M., Abnous, K., 2016. A novel M-shape electrochemical aptasensor for ultrasensitive detection of tetracyclines. Biosens. Bioelectron. 85, 509-514.

Tajik, S., Beitollahi, H., 2019. A sensitive chlorpromazine voltammetric sensor based on graphene oxide modified glassy carbon electrode. Analytical and Bioanalytical Chemistry Research 6 (1), 171-182.

Tajik, S., Beitollahi, H., Biparva, P., 2018. Methyldopa electrochemical sensor based on a glassy carbon electrode modified with $\mathrm{Cu} / \mathrm{TiO} 2$ nanocomposite. J. Serb. Chem. Soc. 83 (7-8), 863-874.

Takahashi, F., Nitta, S., Shimizu, R., Jin, J., 2018. Electrochemiluminescence and voltammetry of tris (2, 2'-bipyridine) ruthenium (II) with amphetamine-type stimulants as coreactants: an application to the discrimination of methamphetamine. Forensic Toxicol. 36 (1), 185-191.

Taleat, Z., Khoshroo, A., Mazloum-Ardakani, M., 2014. Screen-printed electrodes for biosensing: a review (2008-2013). Microchimica Acta 181 (9-10), 865-891.

Tan, W., Donovan, M.J., Jiang, J., 2013. Aptamers from cell-based selection for bioanalytical applications. Chem. Rev. 113 (4), 2842-2862.

Tanaka, K., Ohmor, T., Inoue, T., Seta, S., 1994. Impurity profiling analysis of illicit methamphetamine by capillary gas chromatography. J. Forensic Sci. 39 (2), 500-511.

Vashist, S.K., Zheng, D., Al-Rubeaan, K., Luong, J.H., Sheu, F.-S., 2011. Advances in carbon nanotube based electrochemical sensors for bioanalytical applications. Biotechnol. Adv. 29 (2), 169-188.

Wang, X., Huang, S.-C., Huang, T.-X., Su, H.-S., Zhong, J.-H., Zeng, Z.-C., Li, M.-H., Ren, B., 2017. Tip-enhanced Raman spectroscopy for surfaces and interfaces. Chem. Soc. Rev. 46 (13), 4020-4041.

Wang, Z., Dong, B., Feng, G., Shan, H., Huan, Y., Fei, Q., 2019. Water soluble HeminmPEG-enhanced luminol chemiluminescence for sensitive detection of hydrogen peroxide and glucose. Anal. Sci., 19P150

Watanabe, K., Okada, K., Katsu, T., 1993. Determination of methamphetamine in urine in situ using a methamphetamine-sensitive membrane electrode. Anal. Chim. Acta 274 (1), 59-63.

Wearne, T.A., Cornish, J.L., 2018. A comparison of methamphetamine-induced psychosis and schizophrenia: a review of positive, negative, and cognitive symptomatology. Front. Psychiatr. 9, 491.

Wen, D., Fu, Y., Shi, L., He, C., Dong, L., Zhu, D., He, Q., Cao, H., Cheng, J., 2012. Fine structural tuning of fluorescent copolymer sensors for methamphetamine vapor detection. Sensor. Actuator. B Chem. 168, 283-288.

Weng, S., Dong, R., Zhu, Z., Zhang, D., Zhao, J., Huang, L., Liang, D., 2018. Dynamic 
surface-enhanced Raman spectroscopy and Chemometric methods for fast detection and intelligent identification of methamphetamine and 3, 4Methylenedioxy methamphetamine in human urine. Spectrochim. Acta Mol. Biomol. Spectrosc. 189, 1-7.

Won, S., Hong, R.A., Shohet, R.V., Seto, T.B., Parikh, N.I., 2013. Methamphetamineassociated cardiomyopathy. Clin. Cardiol. 36 (12), 737-742.

Xiao, R., Wang, D., Lin, Z., Qiu, B., Liu, M., Guo, L., Chen, G., 2015. Disassembly of gold nanoparticle dimers for colorimetric detection of ochratoxin A. Analytical Methods 7 (3), 842-845.

Yan, X., Cao, Z., Lau, C., Lu, J., 2010. DNA aptamer folding on magnetic beads for sequential detection of adenosine and cocaine by substrate-resolved chemiluminescence technology. Analyst 135 (9), 2400-2407.

Yarbakht, M., Nikkhah, M., 2016. Unmodified gold nanoparticles as a colorimetric probe for visual methamphetamine detection. J. Exp. Nanosci. 11 (7), 593-601.

Yeh, C.-H., Wang, W.-T., Shen, P.-L., Lin, Y.-C., 2012. A developed competitive immunoassay based on impedance measurements for methamphetamine detection. Microfluid. Nanofluidics 13 (2), 319-329.

Yi, C., Tao, Y., Wang, B., Chen, X., 2005. Electrochemiluminescent determination of methamphetamine based on tris (2, 2'-bipyridine) ruthenium (II) ion- association in organically modified silicate films. Anal. Chim. Acta 541 (1-2), 73-81.

Yu, B., Ge, M., Li, P., Xie, Q., Yang, L., 2019. Development of surface-enhanced Raman spectroscopy application for determination of illicit drugs: towards a practical sensor. Talanta 191, 1-10.

Yu, Y., Xu, W., Fu, Y., Cao, H., He, Q., Cheng, J., 2020. Receptor fluoride fine-tuning of fluorescent polymer probe for highly sensitive fluorescence response of methamphetamine vapor. Dyes Pigments 172, 107852.

Zagatto, E.A.G., Oliveira, C.C. Townshend, A., Worsfold, P., 2011. Flow Analysis with Spectrophotometric and Luminometric Detection. Elsevier.

Zhang, J., Wang, L., Pan, D., Song, S., Boey, F.Y., Zhang, H., Fan, C., 2008. Visual cocaine detection with gold nanoparticles and rationally engineered aptamer structures. Small 4 (8), 1196-1200.

Zhang, L.-Y., Liu, Y.-J., 2014. Label-free amperometric immunosensor based on prussian blue as artificial peroxidase for the detection of methamphetamine. Anal. Chim. Acta 806, 204-209.

Zheng, J., He, L., 2014. Surface-enhanced Raman spectroscopy for the chemical analysis of food. Compr. Rev. Food Sci. Food Saf. 13 (3), 317-328. 